\title{
TERRESTRIAL PLANET FORMATION IN THE $\alpha$ CENTAURI SYSTEM
}

\author{
ELISA V. QUINTANA \\ Space Sciences Division 245-3, NASA Ames Research Center, Moffett Field, CA 94035, \\ and Department of Physics, University of Michigan, Ann Arbor, MI 48109; \\ equintan@pollack.arc.nasa.gov \\ JACK J. LisSAUER AND JOHN E. Chambers \\ Space Sciences Division 245-3, NASA Ames Research Center, Moffett Field, CA 94035 \\ AND \\ Martin J. DUNCAN \\ Department of Physics, Queen's University, Kingston, ON K7L 3N6, Canada \\ Received 2002 February 22; accepted 2002 May 15
}

\begin{abstract}
We examine the late stages of terrestrial planet formation around each star in the $\alpha$ Centauri $\mathrm{A}$ and $\alpha$ Centauri B binary system. Each integration begins with a "bimodal" mass distribution of 14 large embryos embedded in a disk of smaller planetesimals orbiting one of the stars. These initial conditions were chosen because when they are used in simulations about a single star with giant planets, they lead to systems most closely resembling our solar system. However, it is far from certain that such a planetesimal distribution actually occurs either in single or binary star systems. We follow the evolution of the accreting bodies at various values of the inclination of the midplane of the disk relative to the binary orbit for 200 Myr to 1 Gyr. In simulations in which the midplane of the disk was inclined $\leq 30^{\circ}$ relative to the binary orbital plane, three to five terrestrial planets were formed around $\alpha \mathrm{Cen} \mathrm{A}$. When the embryos in the disk were moving retrograde relative to the binary plane, four or five terrestrial planets formed. From two to five planets formed in a disk centered around $\alpha$ Cen $\mathrm{B}$, with $\alpha$ Cen A perturbing the system in the same plane. The aforementioned resulting terrestrial planetary systems are quite similar to those produced by calculations of terrestrial planet growth in the Sun-Jupiter or Sun-Jupiter-Saturn systems. In contrast, terrestrial planet growth around a star lacking both stellar and giant planet companions is slower and extends to larger semimajor axis for the same initial disk of planetary embryos. In systems with the accreting disk initially inclined at $45^{\circ}$ relative to the binary star orbit, two to five planets formed despite the fact that more than half of the disk mass was perturbed into the central star. When the disk was inclined at $60^{\circ}$ to the binary plane, the stability of the planetary embryos decreased dramatically because of larger variations in eccentricity caused by the Kozai resonance, and almost all of the planetary embryos and planetesimals were lost from these systems.
\end{abstract}

Subject headings: binaries: general — planetary systems: formation planetary systems: protoplanetary disks

\section{INTRODUCTION}

The recent detection of more than 80 planets outside of the solar system has provided new insight into current theories on the formation and diversity of planetary systems. Some of these extrasolar planets orbit stars that also possess a stellar companion, e.g., 16 Cygni $\mathrm{B}, \tau$ Bootis, and $55 \rho$ Cancri (Cochran et al. 1997; Butler et al. 1997), confirming that planets can form in binary star systems. However, in each case the stellar separation exceeds $100 \mathrm{AU}$. It is unclear at present whether planets can form in binary star systems with smaller separations between the stars. To date, up to $30 \%$ of the planets discovered outside the solar system are suspected to exist in multiple star systems (Kortenkamp, Wetherill, \& Inaba 2001), although the effect of the stellar companions on the formation of these planets remains unclear.

The radial velocity technique used to discover these planetary companions is able to detect only large (Jupiter-mass) planets, so the existence of Earth-sized planets in binary star systems still remains observationally unconstrained. Since more than half of all stars have at least one stellar companion (Abt 1977), it is worthwhile to examine terrestrial planet growth around one or both stars in a binary system.

The process in which binary star systems are formed is not yet well understood, although current theories suggest that these systems result from the fragmentation of a collapsing molecular cloud core (Bate 2000). Numerical simulations of the collapse of such cores also indicate that disks of material are likely to form (Bodenheimer et al. 2000 and references therein). Material orbiting the binary at distances comparable to the binary semimajor axis, $a_{B}$, will be dynamically unstable on short timescales. However, a circumbinary disk with an inner edge at $\sim 1.8 a_{B}-3 a_{B}$ or beyond can be long lived (Lubow \& Artymowicz 2000). Disks also can persist around the individual components of the binary; for a stellar mass ratio of $3: 1$, a circumprimary disk is stable out to between $0.35 a_{B}$ and $0.5 a_{B}$, while a circumsecondary disk will be truncated between $0.2 a_{B}$ and $0.3 a_{B}$ (Lubow \& Artymowicz 2000). 
Observations of excess radiation from the millimeter range to the near-IR, spectral veiling, Balmer and forbidden emission lines, and polarization indirectly suggest the existence of disk material around one or both components within young binary star systems (Mathieu et al. 2000). The masses of disks around the components of very wide binaries and of circumbinary disks around very close binaries are distributed in a manner similar to the masses of disks around isolated young stars; young binaries with semimajor axes of order a few AU characteristically possess lower mass disks, but many of these disks still have masses comparable to a minimum-mass solar nebula (Mathieu et al. 2000). A small circumstellar disk around one of the components in a binary T Tauri star has been directly imaged by Akeson, Koerner, \& Jensen (1998). Rodriguez et al. (1998) imaged two clearly separated circumstellar disks around the two components of a binary system (which have a binary semimajor axis of $\sim 45 \mathrm{AU}$ ) in L1551. Their observations suggest the presence of circumstellar disks extending out to $\sim 10$ AU from each star with masses of $\sim 0.05 M_{\odot}$.

These observations support the "planetesimal theory" of planet formation (Safronov 1969; Lissauer 1993), in which planets accrete and form from a circumstellar dust disk. In this model, microscopic dust grains first collide and accrete into small (1-10 km diameter) planetesimals, which then collide and accrete one another. Gravity increases planetesimal collision cross sections, and this combined with dynamical friction leads to a "runaway growth" phase, in which the largest bodies accrete material faster than smaller bodies because the largest bodies have substantially larger collision cross sections (Greenberg et al. 1978; Wetherill \& Stewart 1989). Planetary embryos form by this process, and they continue to interact gravitationally and collisionally with one another as well as with smaller bodies within the disk until the remaining material is accreted or dispersed into interstellar space, and the final planets ultimately form on stable widely separated orbits. We wish to learn how the presence of a companion star in such a system affects the planet-forming processes.

Herein, we examine the late stages of planet formation in the $\alpha$ Centauri system, which is composed of a central binary consisting of the G2 star $\alpha$ Cen A $\left(1.1 M_{\odot}\right)$ and the $\mathrm{K} 1$ star $\alpha$ Cen B $\left(0.91 M_{\odot}\right)$. The stars have an orbital semimajor axis of 23.4 AU and an eccentricity of 0.52 . The M5 star $\alpha$ Cen C (Proxima Cen) is thought to orbit this pair but at a very large distance (12,000 AU), and it is neglected in our simulations. Calculations show that luminosity variations on planets in the habitable zones of $\alpha$ Cen A or B resulting from variations in the distance from the companion star would not be substantial during the main-sequence lifetime of the larger star but that when $\alpha$ Cen A becomes a red giant, the habitable zone around $\alpha$ Cen $\mathrm{B}$ will vanish (Hale 1996).

The stability of planets around each star in the $\alpha$ Cen binary system, as well as around the entire system, was examined using direct numerical integrations by Wiegert \& Holman (1997). Their results show that planets can remain stable for several million years for semimajor axes within 3 AU of either $\alpha$ Cen A or $\alpha$ Cen B, or beyond $70 \mathrm{AU}$ of the center of mass of the system, if they are at a low inclination relative to the binary orbital plane. Systems with planets inclined between $60^{\circ}$ and $120^{\circ}$ to the binary plane, however, quickly become unstable. Marzari \& Scholl (2000) studied the evolution of planetesimals perturbed by gas drag in a disk coplanar with the $\alpha$ Cen binary system. Their calculations found that periapse alignment makes it possible for planetesimals to accrete one another within $2 \mathrm{AU}$ of $\alpha$ Cen $\mathrm{A}$, even though epicyclic velocities initially exceed mutual escape speeds. Kortenkamp \& Wetherill (2000) find analogous results for planetesimal growth in the terrestrial planet region around a single star that is orbited by a rapidly formed Jupiter and Saturn. Nonetheless, it is unclear whether or not the distribution of planetary embryos and planetesimals in the terrestrial planet regions around $\alpha$ Cen $\mathrm{A}$ or $\mathrm{B}$ at the beginning of the final phases of planetary growth simulated herein would be similar to that around a single star.

Radial velocity observations by Endl et al. (2001) have excluded the possibility of "51 Peg-type" planets in orbit around either $\alpha$ Cen A or B. These combined with the numerical simulations of Wiegert \& Holman (1997) have also placed upper limits on the mass of planets around each component in $\alpha$ Cen: 2.5 Jupiter masses $\left(M_{\mathrm{J}}\right)$ for planets around $\alpha$ Cen A and 3.5 $M_{\mathrm{J}}$ for planets in orbit around $\alpha$ Cen B. However, these observations were not capable of detecting terrestrial planets. Herein, we examine the late stages of terrestrial planet growth around $\alpha$ Cen $\mathrm{A}$ and $\alpha$ Cen B for various initial inclinations of the circumstellar disk relative to the binary orbital plane.

For our numerical simulations, we assume that planetesimals and larger planetary embryos have already formed from a dust disk around either $\alpha$ Cen A or $\alpha$ Cen B. The calculations of Marzari \& Scholl (2000) referenced above imply that a planetesimal/planetary embryo disk could have formed within the plane of the binary with the stars already in their current orbital configuration. The inclined disks included as initial conditions for some of our simulations may, however, be possible only if the binary star configuration was formed or altered just prior to the epoch at which our integrations begin. Using a newly developed symplectic $N$-body accretion algorithm designed specifically to handle planetary growth around one component in a binary star system (Chambers et al. 2002), we follow the growth of planetary embryos subject to gravitational forces from the central star and the binary companion and to inelastic collisions and gravitational forces between one another. For each simulation, we select the orientation of the disk of planetesimals relative to the binary orbital plane and follow the evolution of the accreting bodies. A binary companion present during the highly dissipative epochs of planetesimal formation and early runaway growth would likely force the planetesimal disk into the plane of the binary orbit. However, because many binary stars may originate as unstable triple star systems, it is also worthwhile to consider the situation when the final stellar configuration is created at a significant inclination to the accreting disk of planetesimals and protoplanets. We examine the effects of chaos in the formation of planetary systems by performing a second set of integrations with a very slight change in the initial conditions of one body. Section 2 explains our numerical model, while $\S 3$ describes the initial conditions of the systems simulated. Section 4 presents our results, and $\S 5$ discusses their implications. Finally, the Appendix presents our results of simulations of planetary accretion around the Sun using the same initial conditions as our $\alpha$ Cen simulations but with no stellar companion (nor giant planets) perturbing the system. 


\section{NUMERICAL MODEL}

To simulate the late stages of planetary accretion in binary star systems, we have incorporated an extra stellar perturbation into the MERCURY5 symplectic hybrid integrator (Chambers 1999), which can also handle close approaches between bodies. Such algorithms, which are based on the $N$-body mapping technique of Wisdom \& Holman (1991), are an order of magnitude faster than conventional integrators and do not produce a buildup of the energy error of a system. We have modified the algorithm to simulate planetary accretion in two binary configurations: the "wide-binary" case, in which planetesimals orbit one star with the stellar companion perturbing the system, and the "close-binary" system, in which the planetesimals orbit the center of mass of the binary system. Multiple tests of the code have been performed, including reproducing the results of the stability simulations of Wiegert \& Holman (1997) and also ensuring the conservation of the Jacobi constant (see Chambers et al. 2002 for details).

Herein, we describe our simulations of the late stages of terrestrial planet formation in the wide-binary $\alpha$ Cen $\mathrm{A}$ and B system. In our model, planets are formed by an accretion process where collisions are taken to be perfectly inelastic. An embryo or planetesimal is removed from the system if ejected beyond $100 \mathrm{AU}$ of the central star. In most of the runs, a body is lost from the system if it falls within $0.1 \mathrm{AU}$ of the primary star, and a 7 day time step is used for these simulations. For systems in which the midplane of the disk began at high inclinations relative to the plane containing $\alpha$ Cen $\mathrm{A}$ and $\mathrm{B}\left(\geq 45^{\circ}\right)$, the Kozai resonance causes large variations in the eccentricity and inclination of the embryos (Innanen et al. 1997; Kozai 1962), and many more bodies have close encounters with the central star. We thus performed some of these simulations a second time but decreased the distance at which bodies are removed to 0.05 AU and the time step of the integration to 1 day. Each system was integrated for times ranging from $200 \mathrm{Myr}$ to 1 Gyr.

\section{INITIAL CONDITIONS}

The initial mass distribution of the circumstellar disk chosen for our model is the same as one of those used in recent simulations by Chambers (2001), which examined terrestrial planet accretion in the solar system with earlyformed Jupiter and Saturn perturbing the system. Of the various disk models examined in that paper, a "bimodal" mass distribution produced planetary configurations most like the terrestrial planets in the solar system. We thus chose to use this model to examine accretion around each component in the $\alpha$ Cen binary system (each of which has comparable mass to the Sun). In this way, we can determine the differences between the outcomes in the $\alpha$ Cen systems relative to those in our solar system simulations, using virtually the same initial disk and the same physical assumptions. In the bimodal model, 14 large bodies (or "embryos") contain half of the disk mass while 140 smaller bodies ("planetesimals") contain the other half. The radii of these bodies are calculated assuming a material density of $3 \mathrm{~g} \mathrm{~cm}^{-3}$. The initial mass of each embryo is $0.0933 M_{\oplus}\left(=2.8 \times 10^{-7} M_{\odot}\right)$, and they are placed between 0.46 and 2.00 AU of the primary star.
The planetesimals begin with a mass of $0.00933 M_{\oplus}$ $\left(=2.8 \times 10^{-8} M_{\odot}\right)$ and range from 0.36 to $2.05 \mathrm{AU}$. Note that the larger embryos are widely spaced, in agreement with the oligarchic growth result of Kokubo \& Ida (1998). All other orbital elements were chosen at random, with the initial eccentricities ranging from 0 to 0.01 and inclinations to the mean plane of the disk ranging between $0^{\circ}$ and 0.5 .

Each integration began with identical disk parameters, with the exception of a small shift in the mean anomaly of one planetesimal in about half of our simulations. Because these $N$-body systems are highly chaotic, i.e., the results are sensitively dependent on the initial conditions of each body in the system, a small shift of even one body can ultimately affect the number of planets formed. We thus used three slightly different sets of initial conditions for the circumstellar disk: the bimodal mass distribution used in Chambers (2001), the same disk but with one planetesimal near $1 \mathrm{AU}$ shifted by $1 \mathrm{~m}$ along its orbit (which will be referred to as the "shifted" case), and the disk with the same planetesimal shifted by $2 \mathrm{~m}$ along its orbit (the "double-shifted" case).

In the majority of our simulations, the circumstellar disk is centered around $\alpha$ Cen A with $\alpha$ Cen B perturbing the system. The initial inclination, $i$, of the midplane of the disk begins at either $0^{\circ}, 15^{\circ}, 30^{\circ}, 45^{\circ}, 60^{\circ}$, or $180^{\circ}$ relative to the plane containing $\alpha$ Cen A and B. The longitude of periastron of the stellar companion is $\varpi_{*}=\omega_{*}+\Omega_{*}$, and we specify the system uniquely by defining the angle $\varpi_{*}-\Omega_{\text {disk }}$ $\left(=180^{\circ}-\omega_{*}\right)$, which is set to either $90^{\circ}, 150^{\circ}$, or $180^{\circ}$ for each run. We also performed a set of integrations with the disk centered around $\alpha$ Cen $\mathrm{B}$, with $\alpha$ Cen $\mathrm{A}$ orbiting the system in the same plane and direction $\left(i=0^{\circ}\right)$. Our final set of runs follow the evolution of an accreting disk centered around the Sun with neither giant planets nor a stellar companion in the system, the results of which are presented in the Appendix.

Our simulations are labeled first by which star the disk is centered around (where "A" refers to accretion around $\alpha$ Cen A and "B" represents systems with the disk orbiting $\alpha$ Cen B). This prefix is then followed by the inclination $i$ of the midplane of the disk of protoplanets relative to the binary plane, and there are typically four integrations for each disk inclination. For our initial set of simulations, the disk of embryos began in the same plane as $\alpha$ Cen A and B $\left(i=0^{\circ}\right)$. The simulations are labeled as follows:

$$
\begin{aligned}
& \text { Ai0_1 } \longrightarrow \varpi_{*}-\Omega_{\text {disk }}=180^{\circ} \\
& \text { Ai0_2 } \longrightarrow \varpi_{*}-\Omega_{\text {disk }}=180^{\circ}, \text { shifted disk } \\
& \text { A } i 0 \_3 \longrightarrow \varpi_{*}-\Omega_{\text {disk }}=180^{\circ}, \text { double-shifted disk } \\
& \text { A } i 0 \_4 \longrightarrow \varpi_{*}-\Omega_{\text {disk }}=150^{\circ}
\end{aligned}
$$

For the remaining simulations (including the disk centered around $\alpha$ Cen B), four runs were performed for each disk inclination (given by $x$ ):

$$
\begin{aligned}
& \text { Aix_1 } \longrightarrow \varpi_{*}-\Omega_{\text {disk }}=180^{\circ} \\
& \text { Aix_2 } \longrightarrow \varpi_{*}-\Omega_{\text {disk }}=180^{\circ}, \text { shifted disk } \\
& \text { Aix_3 } \longrightarrow \varpi_{*}-\Omega_{\text {disk }}=90^{\circ} \\
& \text { Aix_4 } \longrightarrow \varpi_{*}-\Omega_{\text {disk }}=90^{\circ}, \text { shifted disk }
\end{aligned}
$$

The $45^{\circ}$ and $60^{\circ}$ simulations that have a lower value of the distance to the central star at which bodies are removed are labeled $\mathrm{A} i 45 \mathrm{l}$ and $\mathrm{A} i 60 l$. The results are presented in Table 1 and will be described in more detail in the following section. 


\section{TERRESTRIAL PLANET FORMATION IN THE $\alpha$ CENTAURI SYSTEM}

\subsection{From Planetesimals to Planets}

Figure 1 shows the evolution of the disk centered around $\alpha$ Cen A with its midplane initially in the same plane as $\alpha$ Cen B, and with $\varpi_{*}-\Omega_{\text {disk }}=180^{\circ}$ (run Ai0_1). The eccentricities and semimajor axes of all bodies in the disk are shown at various stages in the $200 \mathrm{Myr}$ integration, and the radius of each symbol is proportional to the radius of the body that it represents. Within the first $10 \mathrm{Myr}$, the more massive embryos remain below $e \sim 0.1$ while the planetesimals have a larger variation in their eccentricities (up to $e \sim 0.5$ ). This is the result of the dynamical friction exerted on the large bodies by the smaller planetesimals. The degree of excitation is similar across the disk, and less than $5 \%$ of the initial mass has been removed from the system in the first 20 Myr. By 50 Myr, five remaining embryos have accreted most of the mass, with 10 planetesimals remaining on highly eccentric orbits. These planetesimals are either swept up by the embryos or have collided with $\alpha$ Cen A within 125 Myr. By the end of the $200 \mathrm{Myr}$ integration, $\sim 88 \%$ of the initial mass has been accreted into five terrestrial planets, with the remainder having been removed from the integration because they either escaped the system or wandered too close to the primary star. Three of these bodies have a final mass less than $0.3 M_{\oplus}$, one planet has a mass of $0.6 M_{\oplus}$, and a $0.95 M_{\oplus}$ planet has been formed at $\sim 1.3$ AU. The final planets have semimajor axes that span the entire region occupied by the initial disk.

Figure 2 shows the evolution of a system with the same initial conditions as Figure 1, aside from one planetesimal initially shifted by $1 \mathrm{~m}$ along its orbit (run Ai0_2). This small change in starting conditions causes run Ai0_2 to diverge from run A $i 0 \_1$ (in terms of the amount of mass remaining in the system) within $10^{4} \mathrm{yr}$, although the characteristics of the planetary embryos in the two runs remain qualitatively similar for over $50 \mathrm{Myr}$. In run Ai0_2, six protoplanets have formed within $50 \mathrm{Myr}$, but on closely spaced orbits. Within the following $15 \mathrm{Myr}$, the inner pair of bodies collide and accrete, the next two embryos (near $1 \mathrm{AU}$ ) accrete to form a planet, and the outermost body falls into $\alpha$ Cen A. By the end of the $200 \mathrm{Myr}$ integration, three terrestrial planets have formed comprised of $86 \%$ of the initial mass distribution. Among these, an Earth-mass planet $\left(0.97 M_{\oplus}\right)$ has formed at $\sim 0.9 \mathrm{AU}$, but on a more eccentric orbit $(e \sim 0.1)$ than Earth occupies.

These two examples demonstrate the chaotic nature of $N$-body systems: an initial shift of just $1 \mathrm{~m}$ of one of over 150 bodies in this system resulted in a different number of

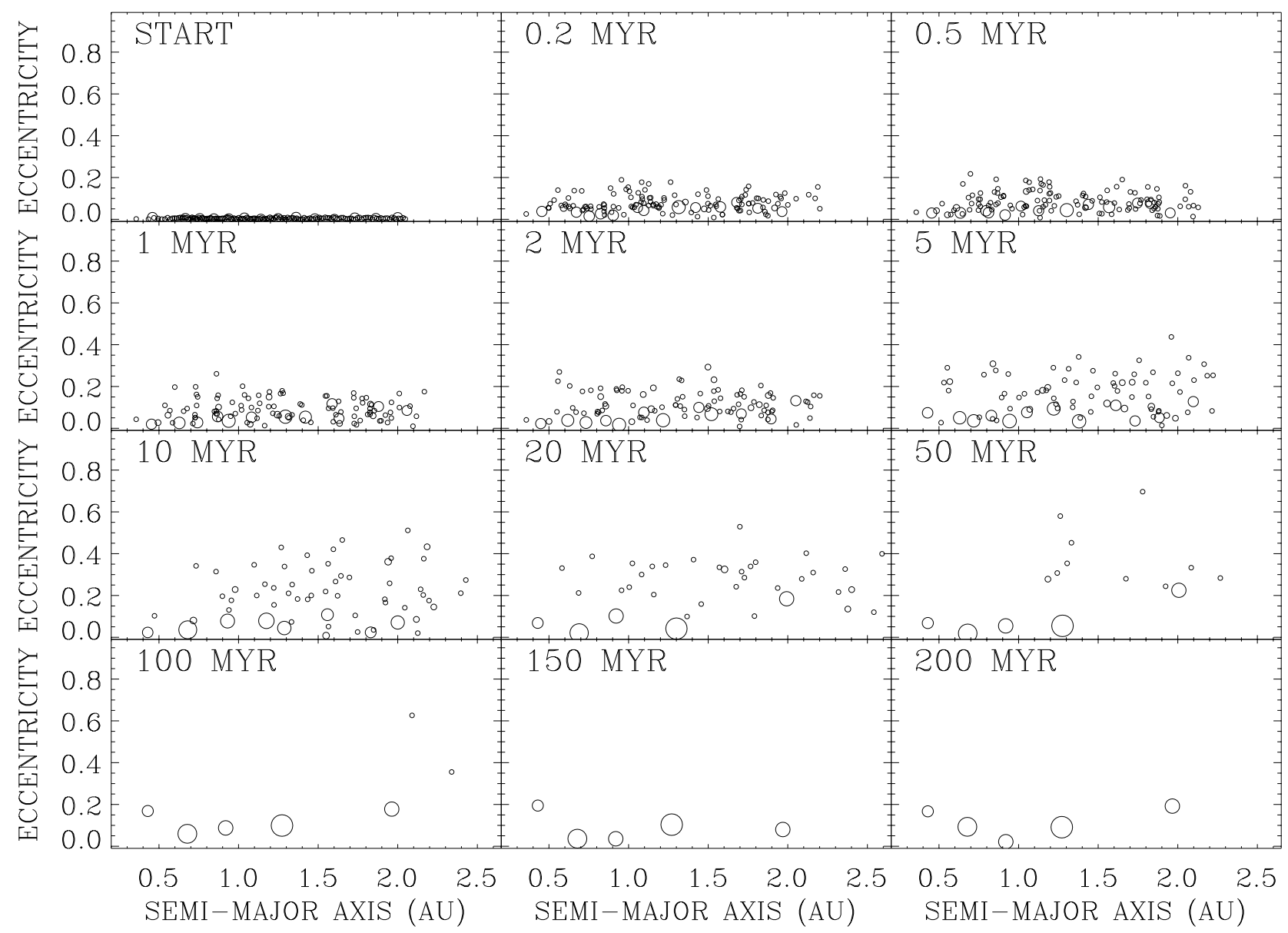

FIG. 1.-Evolution of the circumstellar disk is shown for the Ai0_1 simulation, in which the midplane of the disk coincides with the binary orbital plane. The embryos' and planetesimals' eccentricities are shown as a function of their distance to $\alpha$ Cen A. The radius of each symbol is proportional to the radius of each body that it represents. In this $200 \mathrm{Myr}$ simulation, five terrestrial planets were formed within $2 \mathrm{AU}$ of $\alpha$ Cen $\mathrm{A}$, accreting $~ 88 \%$ of the initial disk mass. Note by comparing the last two panels that mutual perturbations cause planetary eccentricities to vary even after accretion has ended, as is the case in our solar system. 


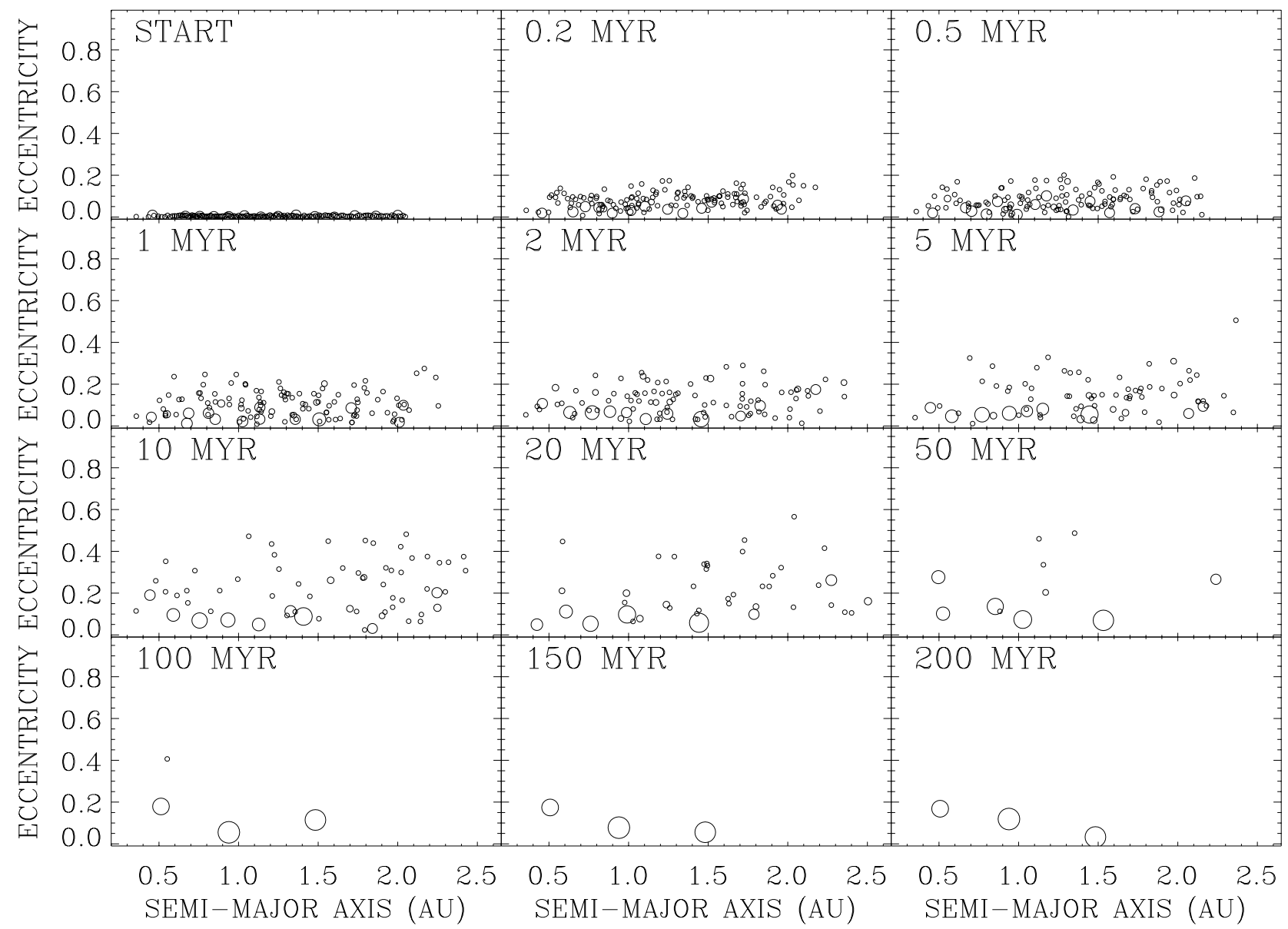

FIG. 2.-Evolution of the disk in system Ai0_2 is shown here. The initial conditions are identical to those used for the run presented in Fig. 1, aside from one planetesimal near $1 \mathrm{AU}$ shifted by $1 \mathrm{~m}$ along its orbit prior to the integration. Because these systems are chaotic, this small change in initial conditions resulted in the formation of three terrestrial planets (composed of $\sim 86 \%$ of the initial mass) compared to the five planets formed in Fig. 1.

final planets. However, each of our integrations in which the disk began inclined $0^{\circ}-30^{\circ}$ relative to the binary plane produced a system of three to five terrestrial planets. The amount of mass lost from the system generally increased slowly with the inclination of the disk relative to the binary plane within this range (see cols. [10] [the percentage of the disk's mass lost into the central star] and [11] [the percentage of the disk's mass ejected from the system] in Table 1, which will be discussed in more detail in $\S 4.2$ ). The radial extent of the disk of material throughout the integrations also varied with the initial inclination. Test particle simulations of Wiegert \& Holman (1997) found that planets can be stable within 2.5-2.7 AU for disk inclinations between $0^{\circ}$ and $30^{\circ}$, within $4.37 \mathrm{AU}$ for retrograde test particles $\left(180^{\circ}\right)$, within 2.07 $\mathrm{AU}$ for $45^{\circ}$ simulations, and within $1.15 \mathrm{AU}$ for $60^{\circ}$ runs.

The circumstellar disk in the $0^{\circ}, 15^{\circ}$, and $30^{\circ}$ simulations typically extended out to $\sim 2.5 \mathrm{AU}$ after of order $10^{7} \mathrm{yr}$ (the initial disk extended out to $2.05 \mathrm{AU}$ ), while the final planets formed in these systems all fell within $\sim 2$ AU. When the planetesimals orbited in the opposite direction to the binary star's orbit $\left(i=180^{\circ}\right)$, the disk extended out to $\sim 3$ AU within 50-100 Myr. Planetesimals in retrograde orbits have shorter encounter times with $\alpha$ Cen B and thus are stable at larger distances from the central star compared with bodies in prograde orbits.
The final planets in these systems all remained within $\sim 2.5$ AU of the central star.

Systems with the midplane of the disk inclined at $45^{\circ}$ tended to produce at least one large planet within $0.5 \mathrm{AU}$ of the central star, with several smaller planets on more eccentric orbits. In these simulations, the effect of the binary companion was stronger, and most of the bodies in the disk were excited up to $e \sim 0.8$ within $100,000 \mathrm{yr}$. The radial extent of the circumstellar disk was less than the lower inclination simulations, with most bodies orbiting within $\sim 2 \mathrm{AU}$ throughout each run. Figure 3 shows the evolution of the masses and eccentricities for all bodies in the disk for a $45^{\circ}$ integration for which the distance at which bodies are removed is $0.05 \mathrm{AU}$ (run Ai45l_1). The stellar companion causes the eccentricities of both planetesimals and planetary embryos to reach high values early in the simulation (i.e., dynamical friction between the planetesimals and embryos is ineffective). More than $40 \%$ of the initial mass in the disk has been perturbed into the star within the first $10 \mathrm{Myr}$. By the end of the simulation, two planets that are more massive than Mercury orbit within $\sim 1.1$ AU while five smaller planets remain in orbit out to $\sim 2 \mathrm{AU}$. Figure 4 shows the masses and inclinations of all bodies in the disk as a function of their semimajor axes at various stages of this simulation. Note that throughout the simulation, most of the bodies remain in highly inclined orbits with $i \sim 45^{\circ}$ relative to the 


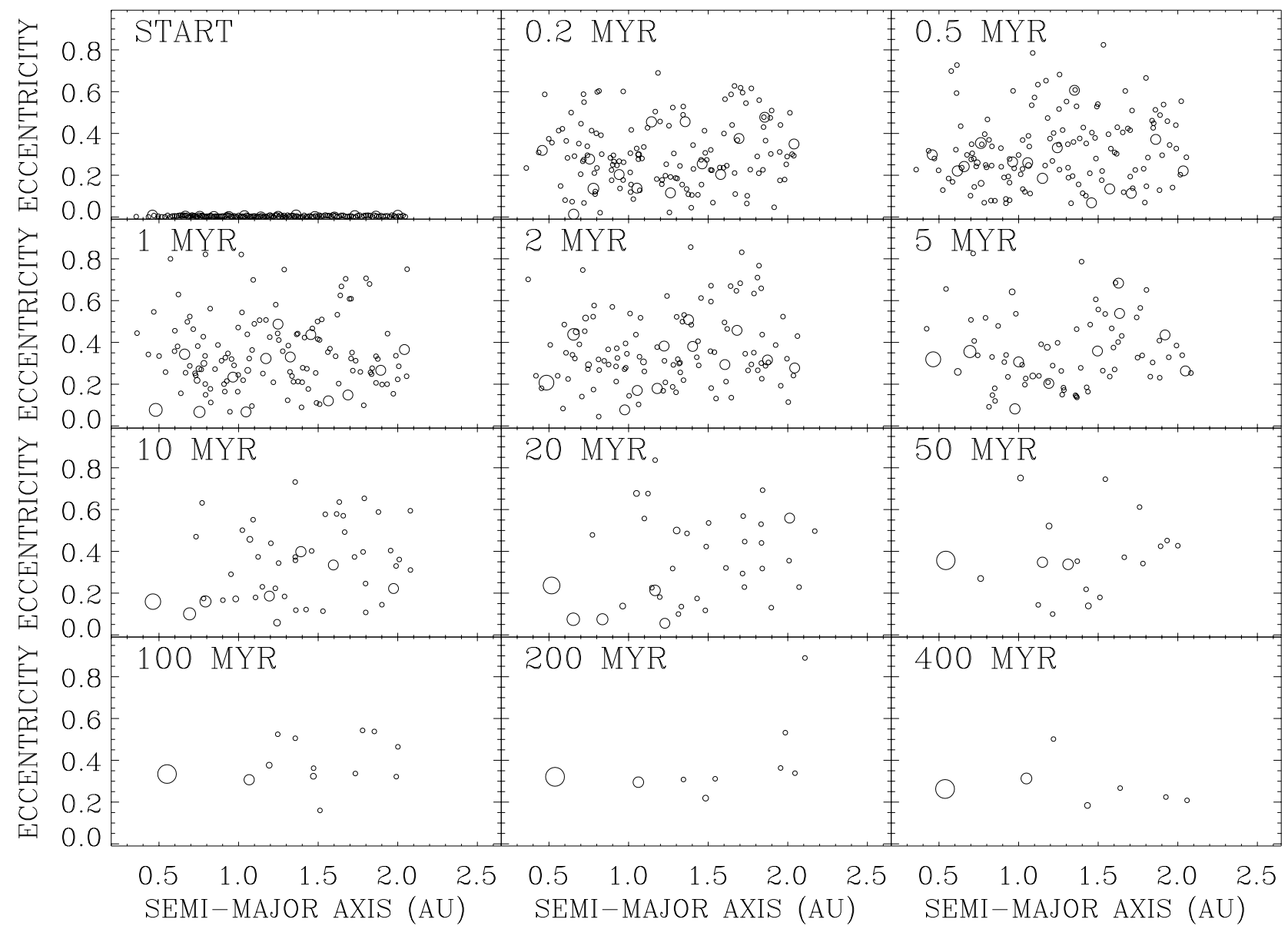

FIG. 3.-Evolution of the A $i 45 l \_1$ system is shown here. In this simulation, the midplane of the disk begins inclined at $45^{\circ}$ relative to the binary orbital plane, and bodies are lost from this system if they come within $0.05 \mathrm{AU}$ of $\alpha \mathrm{Cen} \mathrm{A}$. The radial extent of the disk in this system is more truncated than in the lower inclination systems, and both the embryos' and planetesimals' eccentricities are excited to higher values early in the simulation. The $45^{\circ}$ integrations typically produced a large planet close to the central star, with smaller and more eccentric planets remaining in orbit within 2 AU. Note that the evolution of the disk is shown at different times in comparison with Fig. 1 (for simulations that exceed $200 \mathrm{Myr}$, we omit the panel at $150 \mathrm{Myr}$ and plot the system at $200 \mathrm{Myr}$ and the final integration time).

binary orbital plane. Figure 5 shows the fraction of the mass surviving in the disk as a function of time for the $45^{\circ}$ simulations. On average, $32 \%$ of the initial disk mass was lost from these systems within the first $5 \mathrm{Myr}$. By the end of these integrations, an average of $65 \%$ of the mass had fallen into the central star. Note that the results (Table 1) vary from one integration to another, but the amount of mass loss and the clearing timescale are similar in all cases. Evolution plots similar to Figures 3 and 4 for all of our simulations listed in Tables 1 and 2 can be found in E. V. Quintana (2002, in preparation).

When we increased the disk inclination to $60^{\circ}$, the accretion efficiency decreased dramatically, and the disk was truncated in all of these simulations to $\sim 1.5 \mathrm{AU}$ within 500,000 yr. Figure 6 shows the evolution of the masses and eccentricities of all bodies in a disk in a $60^{\circ}$ inclination run in which the distance at which bodies are removed is 0.05 AU (run Ai60l_1). The perturbative effects of the stellar companion are even more apparent here than in Figure 3, and the eccentricities and inclinations of all bodies are also high early on in the simulation. Most bodies have fallen into the central star within the first few million years, and 95\% of the initial mass has been lost by the end of this simulation. In addition to a greater amount of mass loss, the mass-loss timescale is at least an order of magnitude shorter for the $i=60^{\circ}$ runs than for the $i=45^{\circ}$ integrations. Figure 7 shows the fraction of the surviving mass remaining for these $60^{\circ}$ runs as a function of time. On average, $72.7 \%$ of the initial mass was lost within $500,000 \mathrm{yr}, 88.8 \%$ was lost within 5 Myr, and $94 \%$ was perturbed into $\alpha$ Cen A by the end of these integrations. Decreasing the removal distance from 0.1 to $0.05 \mathrm{AU}$ had only a small (not even statistically significant) effect on the amount of mass lost to the star, so most of the mass would probably be lost even for a more realistic value of $\sim 0.006 \mathrm{AU}$ of $\alpha$ Cen A's collision cross section. In the Ai45l_1 simulation, and in three of the Ai60l runs, the periastron of a single planetesimal in each case fell within $0.1 \mathrm{AU}$ of $\alpha$ Cen A but never fell below $0.05 \mathrm{AU}$ prior to its collision with a larger planetary embryo.

The next section discusses in more detail the properties of all of the planetary systems formed in our $\alpha$ Cen integrations. These results are then compared to a set of $N$-body simulations of terrestrial planet accretion in our solar system performed by Chambers (2001), in which the same initial bimodal mass distribution was used for the circumstellar disk in the Sun-Jupiter-Saturn system. We also compare the final systems of our $\alpha$ Cen integrations to additional simulations that we performed with the same ini- 


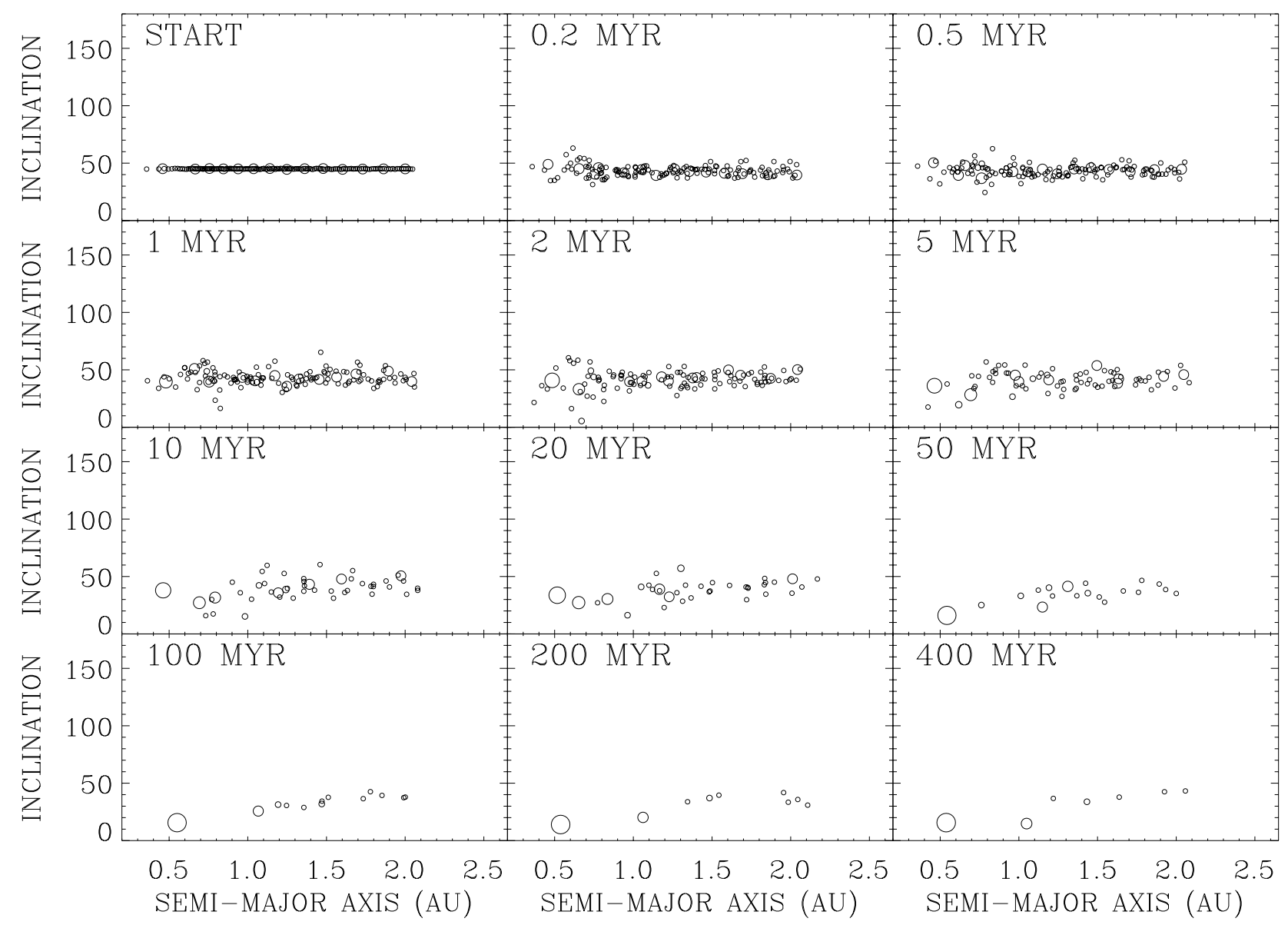

FIG. 4.-Evolution of the masses and inclinations in the Ai45l_1 system are shown here. In this simulation, the midplane of the disk begins inclined at $45^{\circ}$ relative to the binary orbital plane, and bodies are lost from this system if they come within $0.05 \mathrm{AU}$ of $\alpha$ Cen A. Note that the inclinations of most of the bodies remain high throughout the duration of the integration. Similar results were found for all of our simulations.

tial planetesimal/embryo disk orbiting a single star but without giant planets (as described in the Appendix).

\subsection{Final Planetary Systems}

Figures 8 and 9 show the final states of the planetary systems for all of our simulations of terrestrial planet accretion around $\alpha$ Cen $\mathrm{A}$, with the first row (SS) referring to the four terrestrial planets in the solar system. Figure 10 shows the final states for simulations with the disk centered around $\alpha$ $\mathrm{Cen} \mathrm{B}$. The radius of each body is proportional to the radius of the planet that it represents, the horizontal lines through each body indicate the periastron and apastron distances to the central star, the vertical lines represent the inclination relative to the binary orbital plane, while the arrows show the orientation of the final spin axes of each planet (a downward arrow implies retrograde spin).

To examine and quantify the differences of the planetary systems formed in each simulation, we adopt the following set of planetary statistics that were developed to describe the results of planet formation simulations for our solar system (Chambers 2001).

The number of planets, $N_{p}$, that are at least as massive as the planet Mercury $\left(\sim 0.06 M_{\oplus}\right)$.

The number of minor planets, $N_{m}$, remaining at the end of an integration that have not grown as massive as Mercury.
The fraction of the total final remaining mass in the largest planet, $S_{m}$.

An orbital spacing statistic, $S_{s}$, given by

$$
S_{s}=\frac{6}{N_{p}-1}\left(\frac{a_{\max }-a_{\min }}{a_{\max }+a_{\min }}\right)\left(\frac{3 M_{*}}{2 \bar{m}}\right)^{1 / 4},
$$

where $\bar{m}$ is the mean mass of the planets (excluding minor planets), $M_{*}$ is the mass of the central star, and $a_{\max }$ and $a_{\min }$ are the maximum and minimum semimajor axes. For an explanation of the presence of the $\frac{1}{4}$ exponent, see Chambers, Wetherill, \& Boss (1996). Note that minor planets are omitted in the calculation of this statistic because of the nonlinear dependence of this quantity on the planetary masses.

The normalized angular momentum deficit, $S_{d}$, given by

$$
S_{d}=\frac{\sum_{j} m_{j} \sqrt{a_{j}}\left[1-\sqrt{\left(1-e_{j}^{2}\right)} \cos \tilde{i}_{j}\right]}{\sum_{j} m_{j} \sqrt{a_{j}}} .
$$

This statistic measures the fractional difference between the planets' actual orbital angular momenta and the angular momenta that they would have on circular, uninclined orbits with the same semimajor axes. The inclination of each body is measured with respect to the binary orbital plane. 


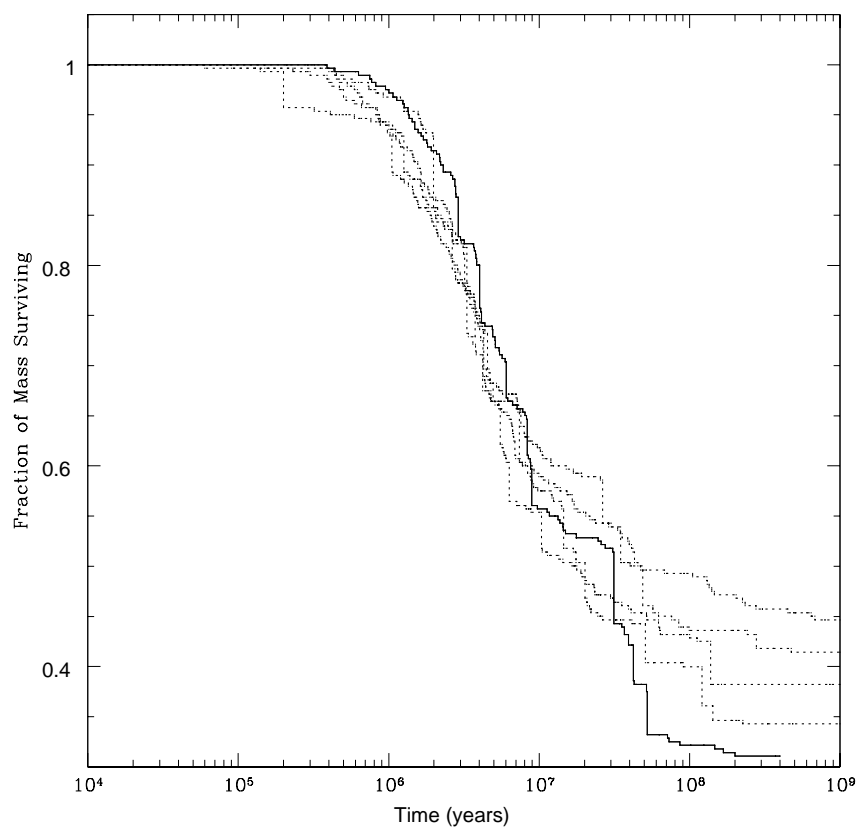

FIG. 5.-Fraction of mass surviving in the disk is shown as a function of time for the $45^{\circ}$ simulations. The dashed lines represent integrations in which bodies were lost from the system if they came within $0.1 \mathrm{AU}$ of the central star, whereas the bold line represents the run in which the minimum distance at which bodies are removed was lowered to $0.05 \mathrm{AU}$. The increase in the stellar companion's initial inclination caused on average $\sim 64 \%$ of the initial disk mass to be lost from the $45^{\circ}$ simulations, all of which was perturbed into the central star.

A mass concentration statistic, $S_{c}$, given by

$$
S_{c}=\max \left\{\frac{\sum_{j} m_{j}}{\sum_{j} m_{j}\left[\log _{10}\left(a / a_{j}\right)\right]^{2}}\right\},
$$

where $m_{j}$ and $a_{j}$ are the mass and semimajor axis of each planet and $S_{c}$ is given by the maximum value of the function in brackets as a function of $a$. This statistic measures the degree to which mass is concentrated in one part of the planetary system.

A radial mixing statistic, $S_{r}$, given by

$$
S_{r}=\left(\sum_{j} \frac{m_{j}\left|a_{\mathrm{init}, j}-a_{\mathrm{fin}, j}\right|}{a_{\mathrm{fin}, j}}\right) / \sum_{j} m_{j},
$$

where $a_{\text {init }}$ and $m$ are the initial semimajor axis and mass of each embryo that becomes incorporated into a final planet and $a_{\text {fin }}$ is the semimajor axis of that planet. This statistic sums the radial migrations of the bodies that form a planet.

Because some of the material in the disk did not accrete into planets in our simulations, we define the following additional set of quantities:

The percentage of the initial disk mass that was lost from the system by falling into the central star, $m_{l_{x}}$.

The percentage of the initial mass that was ejected from the system, $m_{l_{\infty}}$.

The energy per unit mass for the $N$ planets remaining at the end of a simulation, given by

$$
E=\sum_{j} \frac{-G M_{*} m_{j}}{2 a_{j}} / \sum_{j} m_{j},
$$

where $G$ is the gravitational constant, $M_{*}$ is the mass of the central star, $m_{j}$ is the mass of the planet, and $a_{j}$ is the semimajor axis of the planet. This value is normalized by $E_{0}$, the energy (per unit mass) of the system prior to the integration.

The angular momentum of the final $N$ planets (per unit mass), given by

$$
L=\frac{\sum_{j} m_{j}\left[G M_{*} a_{j}\left(1-e_{j}^{2}\right)\right]^{1 / 2}}{\sum_{j} m_{j}},
$$

where $e$ is the planet's eccentricity. This is normalized by $L_{0}$, the angular momentum (per unit mass) of the initial system.

The $Z$-component of angular momentum (per unit mass) relative to the stellar orbit, $L_{Z}$, given by

$$
L_{Z}=L \cos i \text {. }
$$

This value is normalized by $L_{0 Z}$, the initial $Z$-component of angular momentum of the system.

Table 1 gives the values of these statistics for all of our simulations. These will allow us to compare the results of terrestrial planet accretion in the $\alpha$ Cen binary system with the terrestrial planet formation simulations in the SunJupiter-Saturn system of Chambers (2001). We will also compare these to our accretion simulations around the Sun with neither giant planets nor a stellar companion perturbing the system (see Appendix). The first column of Table 1 gives the name of each simulation (as described in $\S 3$ ) for the $\alpha$ Cen system, followed by the four simulations of Chambers (2001) (designated Ch_1-Ch_4) and the solar system planets (labeled MVEM). The next column gives the total integration time, $t$. The systems were integrated for $200 \mathrm{Myr}$ and then continued up to as much as $1 \mathrm{Gyr}$ if it appeared that further accretion was likely to occur (i.e., if bodies were on highly eccentric orbits or continued to interact or have close encounters). Note that in three of the Ai60l runs, the time for the length of the integration is given, followed by the time when a final collision resulted in just one planet remaining in orbit about the central star.

The final numbers of planets and minor planets are given in columns (3) and (4) of Table 1. Of the various initial circumstellar mass distributions simulated in Chambers (2001), the bimodal disk produced final planetary systems around the Sun that were most like those in the solar system, containing three to four planets on widely spaced orbits within $\sim 1.6$ AU. Earth-mass planets were common in these integrations. However, the $e$ and $i$ of many of the planets were generally higher than the terrestrial planets in the solar system, especially for Earth and Venus. Our simulations using this same disk around the Sun, but neglecting giant planets, produced four planets within $2.5 \mathrm{AU}$ in each system and an average of $\sim 12$ minor planets (most of which orbited the Sun beyond 2.5 AU). In all of the $\alpha$ Cen simulations in which the disk was inclined at $\leq 30^{\circ}$ or moved in the retrograde direction $\left(i=180^{\circ}\right)$, three to five planets more massive than Mercury were formed while one to two minor planets remained in orbit in two of the $15^{\circ}$ runs. When the disk was inclined at $45^{\circ}$, two to four planets and zero to five minor planets were formed, although accretion was much less efficient. Most of the mass was perturbed into the central star when the inclination of the disk was raised to $60^{\circ}$, and only one planet more massive than Mercury was formed in all but one simulation, while zero to two minor planets remained in each system. Note that many of the col- 


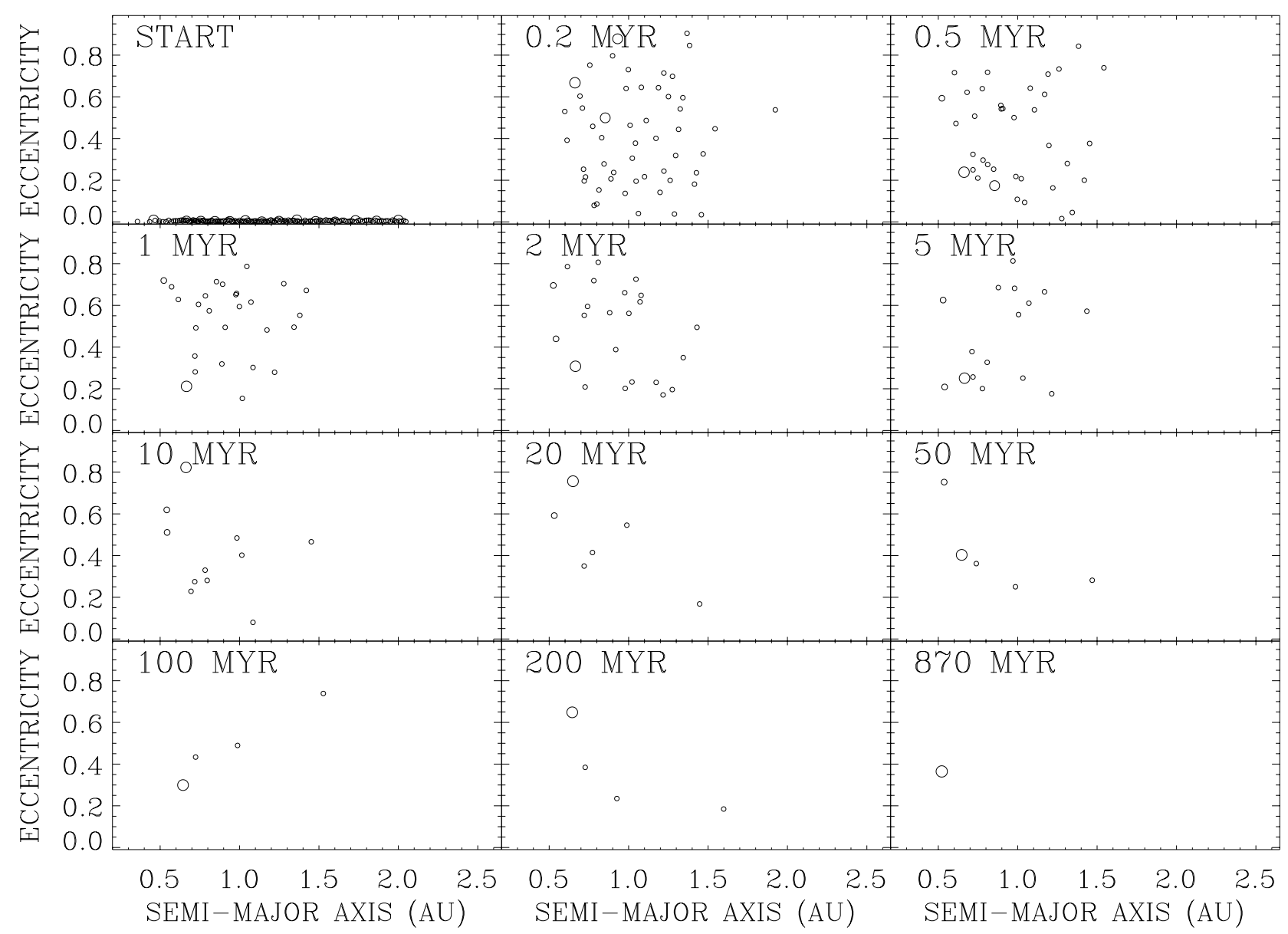

FIG. 6. - Evolution of the A $i 60 l \_1$ system is shown here. In this simulation, the midplane of the disk begins at $i=60^{\circ}$ and the distance at which bodies are removed from the system is 0.05 AU. The binary companion's high initial inclination causes large variations in the eccentricity of each planetesimal and embryo, and most of the mass is perturbed into the central star within the first few million years.

lisions in the high-inclination systems occurred at relative velocities much higher than the mutual escape speeds of the bodies involved. Had we accounted for fragmentation, inclinations and eccentricities might have been damped and the final systems could have differed significantly from the ones produced in our integrations. In our simulations with the disk centered around $\alpha \mathrm{Cen} \mathrm{B}$, two to four terrestrial planets and zero to one minor planets were produced.

The fraction of the total final remaining mass in the largest body, $S_{m}$, is given in column (5) of Table 1. For our solar system, the Earth is composed of slightly more than half $(50.9 \%)$ of the total mass of the four terrestrial planets. The accretion simulations in the Sun-Jupiter-Saturn system, and also the Sun-only simulations, produced on average systems with $\sim 40 \%$ of the total mass in the largest planet. In our integrations with the disk inclined at $0^{\circ}$ and $180^{\circ}, \sim 40 \%$ of the mass also remained in the largest final planet. Our $15^{\circ}$ and $30^{\circ} \alpha$ Cen integrations produced final systems with $S_{m}$ closer to the actual solar system terrestrial planets $(\sim 50 \%$ and $\sim 44 \%$, respectively). The simulations with the disk inclined at $45^{\circ}$, however, tended to produce one large planet close to the central star with much smaller bodies remaining at higher semimajor axes and eccentricities $\left(S_{m} \sim 0.77\right)$. The tendency for more inclined systems to produce a larger planet closer to the star is a consequence of two factors. First, the higher the inclination of a given disk, the smaller is the component of total angular momentum normal to the binary plane. Since that component is largely preserved during the evolution (see the final column in Table 1), initially more inclined disks will tend to produce more compact final systems. Second, for an excited population, the enhanced probability of collisions near pericenter favors the growth of a large embryo close to the central star. The $S_{m}$ value was also high for all of the $60^{\circ}$ runs (an average of $S_{m}=0.94$ ) and for the accretion simulations around $\alpha$ Cen B $\left(S_{m}=0.63\right)$. Thus, there is a general trend that the more strongly perturbed disks tend to produce systems with a larger fraction of mass contained in the biggest terrestrial planet. This result may not hold, however, if fragmentation in high-velocity collisions is taken into account.

The orbital-spacing statistic, $S_{s}$, gives a measure of how widely spaced the final planets are. The solar system's terrestrial planets have a value of $S_{s}=37.7$, while the simulations of Chambers (2001) have an average $S_{s}$ of 36.5. The accretion simulations around the Sun with neither a binary companion nor giant planets in the system (cf. Appendix) produced systems with a comparable average value of $S_{s}=38.2$. Because this statistic has a nonlinear dependence on the mass of the planets $\left(S_{s} \propto m^{-1 / 4}\right)$, we neglect the minor planets because of their small masses. All of the $\alpha$ Cen A simulations with the disk inclined $\leq 30^{\circ}$ produced systems with an average value of $S_{s}$ in the range $35-45$. The 


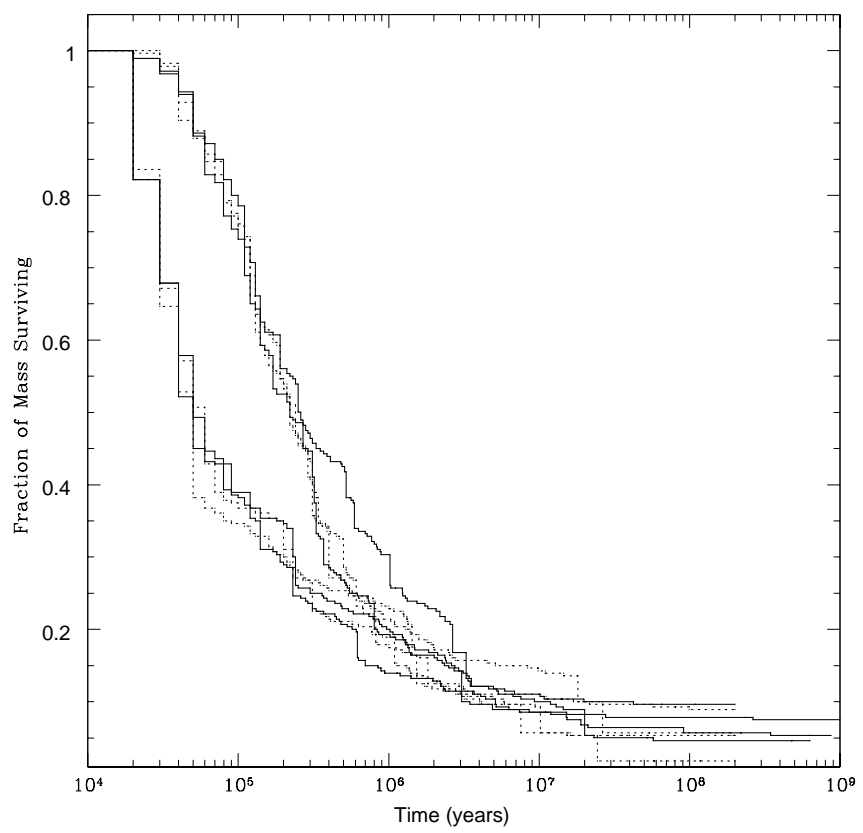

FIG. 7.-Fraction of mass surviving in the disk is shown as a function of time for the $60^{\circ}$ integrations. The dashed lines represent simulations in which the minimum periastron distance at which bodies are removed is 0.1 AU from the central star, and the bold lines represent runs in which this distance was lowered to $0.05 \mathrm{AU}$. The curves fall into two groups, with the four runs having $\varpi_{*}-\Omega_{\text {disk }}=180^{\circ}$ initially losing mass substantially faster than those with $\varpi_{*}-\Omega_{\text {disk }}=90^{\circ}$ because of a larger oscillation in the first group associated with the Kozai effect (Kozai 1962). No systematic difference is seen at later times, nor is a difference depending on removal distance apparent at any time. On average, 94\% of the initial disk mass has fallen into $\alpha$ Cen $\mathrm{A}$ by the end of the $60^{\circ}$ integrations. Note that the timescale for which the fraction of surviving mass falls to half of its initial value for the $60^{\circ}$ runs averages $\sim 1.4 \times 10^{5} \mathrm{yr}$, whereas this timescale averages $\sim 28 \times 10^{6} \mathrm{yr}$ for the $45^{\circ}$ simulations. $45^{\circ}$ final systems, however, are more widely spaced and have a higher average value of $S_{s} \sim 67$. In the $60^{\circ}$ simulations, at most one planet more massive than Mercury remains in each system, so this statistic is not included for these runs. In several $60^{\circ}$ simulations, two bodies remain in crossing orbits for hundreds of millions of years. In the A $i 60 l \_3$ simulation, for example, just two planets remain in the system after 57 Myr. Despite their proximity, these bodies do not collide for an additional $400 \mathrm{Myr}$ of the integration. This is consistent with the expected time required for these two bodies to collide, calculated according to the formulas given in Öpik (1951), which is $\sim 10^{9} \mathrm{yr}$.

The normalized angular momentum deficit, $S_{d}$, is dependent on each planet's mass, semimajor axis, eccentricity, and inclination and is a measure of the orbital excitation of the system. The solar system terrestrial planets have an average (over Myr time spans) value of $S_{d}=0.0018$. The accretion simulations around the Sun with the giant planets perturbing the system produced values of $S_{d}$ more than 3 times higher than the actual solar system $\left(S_{d}=0.0069\right.$, where the inclination in this case is measured with respect to the Sun-Jupiter orbital plane). The Sun-only simulations resulted in even more excited systems $\left(S_{d}=0.0318\right)$, which is attributed primarily to minor planets on very eccentric orbits at large semimajor axes that would most likely be lost if giant planets were also in orbit. All of the planar simulations with the disk around either $\alpha$ Cen A or B had similar $S_{d}$ values as the simulations of accretion in the Sun-JupiterSaturn system, and $S_{d}$ increased as the initial inclination of the accreting disk was increased, as expected.

The mass concentration statistic, $S_{c}$, which is a function of the mass and semimajor axes of the planets, gives a measure of the degree to which mass is concentrated in one part of a final planetary system. In the case of the four terrestrial planets of the solar system, most of the mass is in Earth and

TABLE 1

Statistics for Final Planetary Systems

\begin{tabular}{|c|c|c|c|c|c|c|c|c|c|c|c|c|c|}
\hline $\begin{array}{l}\text { Run } \\
\text { (1) }\end{array}$ & $\begin{array}{c}t \\
\text { (Myr) } \\
(2)\end{array}$ & $\begin{array}{l}N_{p} \\
(3)\end{array}$ & $\begin{array}{l}N_{m} \\
(4)\end{array}$ & $\begin{array}{l}S_{m} \\
(5)\end{array}$ & $\begin{array}{l}S_{s} \\
(6)\end{array}$ & $\begin{array}{l}S_{d} \\
(7)\end{array}$ & $\begin{array}{l}S_{c} \\
(8)\end{array}$ & $\begin{array}{l}S_{r} \\
(9)\end{array}$ & $\begin{array}{l}m_{l_{*}} \\
(\%) \\
(10)\end{array}$ & $\begin{array}{l}m_{l_{\infty}} \\
(\%) \\
(11)\end{array}$ & $\begin{array}{c}E / E_{0} \\
(12)\end{array}$ & $\begin{array}{c}L / L_{0} \\
(13)\end{array}$ & $\begin{array}{c}L_{Z} / L_{Z_{0}} \\
\quad(14)\end{array}$ \\
\hline Ai0_1 .............. & 200 & 5 & 0 & 0.415 & 31.1 & 0.0049 & 32.3 & 0.233 & 11.42 & 0.714 & 1.11 & 0.95 & 0.95 \\
\hline $\mathrm{A} i 0 \_2 \ldots \ldots \ldots \ldots . . . .$. & 200 & 3 & 0 & 0.427 & 42.1 & 0.0082 & 35.0 & 0.323 & 13.22 & 0.714 & 1.14 & 0.93 & 0.92 \\
\hline Ai0_3 .............. & 200 & 5 & 0 & 0.337 & 30.3 & 0.0043 & 30.0 & 0.293 & 10.70 & 0.357 & 1.11 & 0.95 & 0.95 \\
\hline Ai0_4 f.............. & 200 & 4 & 0 & 0.420 & 34.3 & 0.0036 & 32.4 & 0.353 & 11.05 & 1.428 & 1.12 & 0.94 & 0.94 \\
\hline A $i 0 \_$ave............ & 200.0 & 4.25 & 0.00 & 0.400 & 34.5 & 0.0053 & 32.4 & 0.301 & 11.60 & 0.803 & 1.12 & 0.94 & 0.94 \\
\hline A $i 15 \_1 \ldots \ldots \ldots \ldots$ & 1000 & 3 & 2 & 0.457 & 40.7 & 0.0081 & 36.5 & 0.323 & 13.20 & 0.000 & 1.21 & 0.90 & 0.93 \\
\hline A $i 15 \_2 \ldots \ldots \ldots \ldots . .$. & 500 & 3 & 1 & 0.514 & 41.9 & 0.0038 & 46.7 & 0.323 & 25.36 & 0.357 & 1.35 & 0.84 & 0.87 \\
\hline A $i 15 \_3 \ldots \ldots \ldots \ldots . .$. & 500 & 4 & 0 & 0.520 & 39.1 & 0.0076 & 32.1 & 0.482 & 19.28 & 0.357 & 1.27 & 0.88 & 0.91 \\
\hline A $i 15 \_4 \ldots \ldots \ldots \ldots$ & 500 & 3 & 0 & 0.498 & 40.2 & 0.0069 & 31.3 & 0.360 & 15.35 & 0.000 & 1.21 & 0.90 & 0.93 \\
\hline A $i 15$ ave.......... & 625.0 & 3.25 & 0.75 & 0.497 & 40.5 & 0.0066 & 36.7 & 0.372 & 18.30 & 0.179 & 1.26 & 0.88 & 0.91 \\
\hline Ai30_1 .............. & 500 & 4 & 0 & 0.335 & 39.7 & 0.0221 & 23.9 & 0.387 & 23.91 & 0.357 & 1.39 & 0.86 & 0.97 \\
\hline Ai30_2 ............. & 1000 & 4 & 0 & 0.336 & 40.1 & 0.0341 & 22.1 & 0.431 & 16.79 & 0.357 & 1.41 & 0.85 & 0.96 \\
\hline Ai30_3 ............. & 1000 & 3 & 0 & 0.419 & 47.1 & 0.0243 & 26.2 & 0.462 & 24.99 & 0.000 & 1.48 & 0.82 & 0.94 \\
\hline Ai30_4 ............. & 500 & 3 & 0 & 0.659 & 53.3 & 0.0129 & 32.5 & 0.578 & 24.63 & 0.357 & 1.56 & 0.79 & 0.91 \\
\hline Ai30_ave.......... & 625.0 & 3.50 & 0.00 & 0.437 & 45.1 & 0.0234 & 26.2 & 0.465 & 22.58 & 0.268 & 1.46 & 0.83 & 0.95 \\
\hline $\mathrm{A} i 45 \_1 \ldots \ldots \ldots \ldots$ & 1000 & 2 & 0 & 0.808 & 91.8 & 0.0363 & 28.0 & 0.975 & 55.34 & 0.000 & 2.12 & 0.68 & 0.94 \\
\hline A $i 45 \_2 \ldots \ldots \ldots \ldots$ & 1000 & 4 & 1 & 0.673 & 49.8 & 0.1267 & 13.1 & 0.695 & 61.77 & 0.000 & 1.94 & 0.75 & 0.95 \\
\hline $\mathrm{A} i 45 \_3 \ldots \ldots \ldots \ldots$ & 1000 & 2 & 1 & 0.750 & 72.7 & 0.0840 & 41.5 & 0.732 & 65.71 & 0.000 & 1.76 & 0.74 & 0.96 \\
\hline A $i 45 \_4 \ldots \ldots \ldots \ldots$ & 1000 & 3 & 2 & 0.759 & 55.5 & 0.0606 & 24.6 & 1.030 & 58.55 & 0.000 & 2.40 & 0.65 & 0.87 \\
\hline A $i 45$ ave.......... & 1000.0 & 2.75 & 1.00 & 0.748 & 67.5 & 0.0769 & 26.8 & 0.858 & 60.34 & 0.000 & 2.06 & 0.71 & 0.93 \\
\hline A $i 45 l \_1 \ldots \ldots . . . .$. & 400 & 2 & 5 & 0.782 & 65.8 & 0.0851 & 44.4 & 0.678 & 67.85 & 0.000 & 1.70 & 0.72 & 0.97 \\
\hline Ai60_1 ............. & 200 & 0 & 2 & 0.800 & $\ldots$ & 0.2889 & 32.5 & $\infty$ & 98.20 & 0.000 & 2.73 & 0.50 & 0.85 \\
\hline A $i 60 \_2 \ldots \ldots \ldots \ldots . . .$. & 200 & 1 & 2 & 0.920 & $\ldots$ & 0.3456 & 23.8 & 0.438 & 91.08 & 0.000 & 2.39 & 0.54 & 0.85 \\
\hline A $i 60 \_3 \ldots \ldots \ldots \ldots . . .$. & 200 & 1 & 1 & 0.933 & $\ldots$ & 0.4408 & 131.1 & 0.385 & 94.64 & 0.000 & 1.55 & 0.68 & 0.86 \\
\hline
\end{tabular}


TABLE 1-Continued

\begin{tabular}{|c|c|c|c|c|c|c|c|c|c|c|c|c|c|}
\hline $\begin{array}{l}\text { Run } \\
\text { (1) }\end{array}$ & $\begin{array}{c}t \\
(\mathrm{Myr}) \\
(2)\end{array}$ & $\begin{array}{l}N_{p} \\
\text { (3) }\end{array}$ & $\begin{array}{l}N_{m} \\
\text { (4) }\end{array}$ & $\begin{array}{l}S_{m} \\
(5)\end{array}$ & $\begin{array}{c}S_{s} \\
(6)\end{array}$ & $\begin{array}{l}S_{d} \\
(7)\end{array}$ & $\begin{array}{l}S_{c} \\
(8)\end{array}$ & $\begin{array}{l}S_{r} \\
(9)\end{array}$ & $\begin{array}{l}m_{l_{*}} \\
(\%) \\
(10)\end{array}$ & $\begin{array}{l}m_{l_{\infty}} \\
(\%) \\
(11)\end{array}$ & $\begin{array}{c}E / E_{0} \\
(12)\end{array}$ & $\begin{array}{c}L / L_{0} \\
(13)\end{array}$ & $\begin{array}{c}L_{Z} / L_{Z_{0}} \\
\quad \text { (14) }\end{array}$ \\
\hline Ai60_4 .............. & 200 & 1 & 1 & 0.933 & $\ldots$ & 0.2367 & 49.6 & 0.698 & 94.64 & 0.000 & 1.87 & 0.69 & 1.09 \\
\hline Ai60_ave........... & 200.0 & 0.75 & 1.50 & 0.897 & $\ldots$ & 0.3280 & 59.3 & 0.507 & 94.64 & 0.000 & 2.14 & 0.60 & 0.91 \\
\hline Ai60l_1 …............ & 870 (636) & 1 & 0 & 1.000 & $\ldots$ & 0.4079 & $\ldots$ & 0.386 & 94.64 & 0.000 & 1.98 & 0.61 & 0.79 \\
\hline Ai60l_2 .............. & $1000(264)$ & 1 & 0 & 1.000 & $\ldots$ & 0.4114 & $\ldots$ & 0.299 & 92.50 & 0.000 & 1.69 & 0.61 & 0.85 \\
\hline A $i 60 l \_3 \ldots \ldots \ldots \ldots . . .$. & $630(477)$ & 1 & 0 & 1.000 & $\ldots$ & 0.4365 & $\ldots$ & 0.259 & 95.37 & 0.000 & 1.75 & 0.69 & 0.80 \\
\hline Ai60l_4 .............. & 200 & 1 & 1 & 0.963 & $\ldots$ & 0.1756 & 65.0 & 1.749 & 90.36 & 0.000 & 3.45 & 0.51 & 0.86 \\
\hline 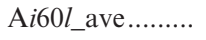 & 397.5 & 1.00 & 0.25 & 0.991 & $\ldots$ & 0.3579 & 65.0 & & 93.22 & 0.000 & 2.22 & 0.61 & 0.83 \\
\hline A $i 180 \_1 \ldots \ldots \ldots . .$. & 500 & 5 & 0 & 0.291 & 30.5 & 1.9972 & 27.4 & 0.313 & 3.92 & 3.928 & 1.10 & 0.96 & 0.96 \\
\hline A $i 180 \_2 \ldots \ldots \ldots . .$. & 200 & 4 & 0 & 0.490 & 36.9 & 1.9958 & 26.1 & 0.280 & 3.55 & 4.642 & 1.10 & 0.96 & 0.96 \\
\hline A $i 180 \_3 \ldots \ldots \ldots . .$. & 1000 & 4 & 0 & 0.457 & 34.7 & 1.9952 & 28.4 & 0.259 & 5.33 & 3.928 & 1.10 & 0.95 & 0.95 \\
\hline A $i 180 \_4 \ldots \ldots \ldots . .$. & 200 & 5 & 0 & 0.336 & 32.0 & 1.9967 & 31.2 & 0.253 & 6.79 & 3.928 & 1.10 & 0.95 & 0.95 \\
\hline A $i 180 \_$ave ......... & 475.0 & 4.50 & 0.00 & 0.394 & 33.5 & 1.9962 & 28.3 & 0.276 & 4.90 & 4.107 & 1.10 & 0.96 & 0.96 \\
\hline Bi0_1 .................. & 200 & 4 & 1 & 0.473 & 34.7 & 0.0053 & 32.8 & 0.314 & 17.15 & 2.142 & 1.18 & 0.92 & 0.91 \\
\hline $\mathrm{B} i 0 \_2 \ldots \ldots \ldots \ldots . . . .$. & 200 & 3 & 1 & 0.695 & 35.9 & 0.0041 & 52.5 & 0.335 & 23.56 & 1.428 & 1.24 & 0.88 & 0.87 \\
\hline $\mathrm{B} i 0 \_3 \ldots \ldots \ldots \ldots . . .$. & 200 & 2 & 0 & 0.695 & 49.7 & 0.0042 & 64.8 & 0.471 & 31.79 & 1.428 & 1.35 & 0.83 & 0.83 \\
\hline $\mathrm{Bi} 0 \_4 \ldots \ldots \ldots \ldots . . . . .$. & 200 & 3 & 0 & 0.643 & 41.5 & 0.0057 & 67.6 & 0.338 & 29.29 & 0.714 & 1.30 & 0.85 & 0.84 \\
\hline Bi0_ave ............ & 200.0 & 3.00 & 0.50 & 0.627 & 40.5 & 0.0048 & 54.4 & 0.365 & 25.45 & 1.428 & 1.27 & 0.87 & 0.86 \\
\hline Ch_1 .................. & 200 & 4 & 0 & 0.335 & 31.3 & 0.0062 & 37.3 & 0.275 & 17.12 & 0.71 & 1.17 & 0.91 & 0.91 \\
\hline 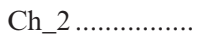 & 150 & 4 & 0 & 0.326 & 33.7 & 0.0105 & 35.0 & 0.262 & 18.55 & 1.43 & 1.19 & 0.91 & 0.90 \\
\hline Ch_3 ................... & 200 & 4 & 0 & 0.493 & 36.4 & 0.0052 & 34.8 & 0.263 & 18.93 & 3.57 & 1.23 & 0.90 & 0.90 \\
\hline Ch_4 .................. & 200 & 3 & 0 & 0.471 & 44.7 & 0.0057 & 32.4 & 0.290 & 24.63 & 0.36 & 1.26 & 0.89 & 0.88 \\
\hline $\mathrm{Ch}$ (ave) .............. & 187.5 & 3.75 & 0.00 & 0.406 & 36.5 & 0.0069 & 34.9 & 0.273 & 19.81 & 1.52 & 1.21 & 0.90 & 0.90 \\
\hline MVEM ................ & $\ldots$ & 4 & 0 & 0.509 & 37.7 & 0.0018 & 89.9 & $\ldots$ & $\ldots$ & $\ldots$ & $\ldots$ & $\ldots$ & $\ldots$ \\
\hline
\end{tabular}

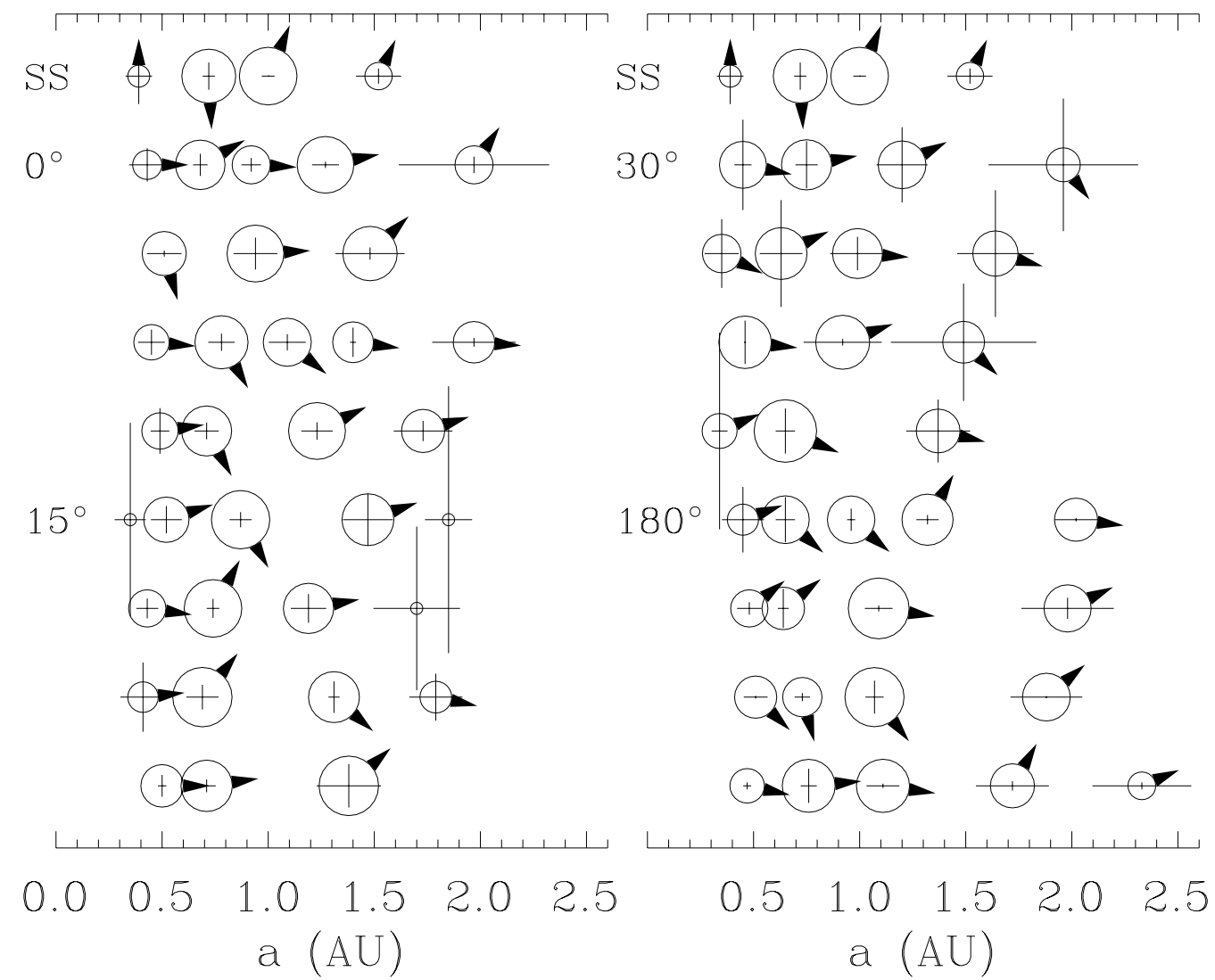

FIG. 8. - Final planetary states for the solar system and our simulations with the midplane of the disk inclined at $0^{\circ}, 15^{\circ}, 30^{\circ}$, and $180^{\circ}$ to the binary orbital plane, and centered around $\alpha \mathrm{Cen} \mathrm{A}$. The radius of each symbol is proportional to the radius of the planet that it represents, the horizontal line through each body signifies the periastron and apastron distances, the vertical line represents the inclination of the planet relative to the binary orbital plane, and the arrow indicates the final spin orientation of each planet. Note that the inclinations of the solar system planets are measured with respect to the invariable plane, and the inclinations for the $180^{\circ}$ runs are subtracted from $180^{\circ}$. In addition, the final spin orientation of planetesimals that did not accrete any material but remained in the system are unknown. In these simulations, three to five terrestrial planets formed within $\sim 2.5 \mathrm{AU}$, most of them on roughly circular and on roughly coplanar orbits. 
Venus, with $S_{c}=89.9$. The simulations of Chambers (2001) and the Sun-only integrations produced systems with much lower (average) values of 34.9 and 15.9, respectively. The $\alpha$ Cen simulations with the disk centered around $\alpha$ Cen A inclined at $\leq 45^{\circ}$ resulted in average values of $S_{c}$ between 26 and 45 , whereas the $60^{\circ}$ runs and the $\alpha$ Cen B systems had higher average values between $S_{c}=54$ and 65 .

The radial mixing statistic, $S_{r}$, is a measure of the radial migrations of each body that makes up a final planet and is weighted according to the embryo's mass. This value cannot be compared to the solar system, but the systems simulated in the Sun-Jupiter-Saturn planets had an average $S_{r}$ of 0.273 . The average value was higher for the Sun-only systems $(0.360)$. The $\alpha$ Cen simulations with the disk inclination $\leq 15^{\circ}$ all had average values of $S_{r}$ comparable to the simulations around the Sun, with $S_{r}$ between 0.25 and 0.37 . This value increased to 0.47 for the $30^{\circ}$ runs and ranged from 0.67 to 0.86 for the integrations with the disk inclined $\geq 45^{\circ}$.

The percentage of the initial mass in the disk that was lost from the system by falling into the central star, $m_{l_{*}}$, is clearly a function of the disk's inclination. The nearly planar simulations had the least amount of disk mass loss (with a prograde disk losing an average of $\sim 11.6 \%$ of the initial mass and the retrograde disks losing an average of $4.9 \%$ ). This percentage increased with increasing disk inclinations, but at least $\sim 75 \%$ of the initial mass remained in all systems with $i \leq 30^{\circ}$ (including the disk centered around $\alpha$ Cen B). More than $60 \%$ of the initial mass was perturbed into the central star in the $45^{\circ}$ runs, and over $90 \%$ of the mass was lost in the $60^{\circ}$ simulations. The majority of bodies that were lost from the systems were perturbed into the central star, so our placement of the inner disk boundary at 0.1 or $0.05 \mathrm{AU}$ rather than at the stellar photosphere of $\lesssim 0.01 \mathrm{AU}$ implies that our simulations overestimate the amount of mass loss; however, the similarity between the amounts of mass lost in the runs with the boundary at $0.05 \mathrm{AU}$ to that in runs with the inner boundary at $0.1 \mathrm{AU}$ suggests that this error is small. In some cases, minor planets were ejected from the system, but in the higher inclination simulations all of the mass that was lost was rapidly perturbed into the star and no bodies were ejected into interstellar space.

In the planar simulations with the disk centered around $\alpha$ Cen A, the (magnitude of the negative total) energy per unit mass of the final planetary systems increased by $\sim 10 \%$ relative to the energy of the initial mass distribution. A 25\% increase of this energy for both the $15^{\circ}$ simulations and the $\alpha$ Cen B runs is comparable to the simulations of the SunJupiter-Saturn system. This energy increased by more than $100 \%$ for many systems with initial disk inclinations $\geq 45^{\circ}$, as most of the final bodies orbit closer to the central star. The angular momentum of the final planetary systems only slightly decreased for the planar simulations while continuing to decrease as the stellar companion inclination was increased. For the $60^{\circ}$ runs, an average of $40 \%$ of the specific angular momentum was lost from these systems. The Zcomponent of specific angular momentum, however, typically decreased by only $\sim 10 \%$ in all of the integrations.

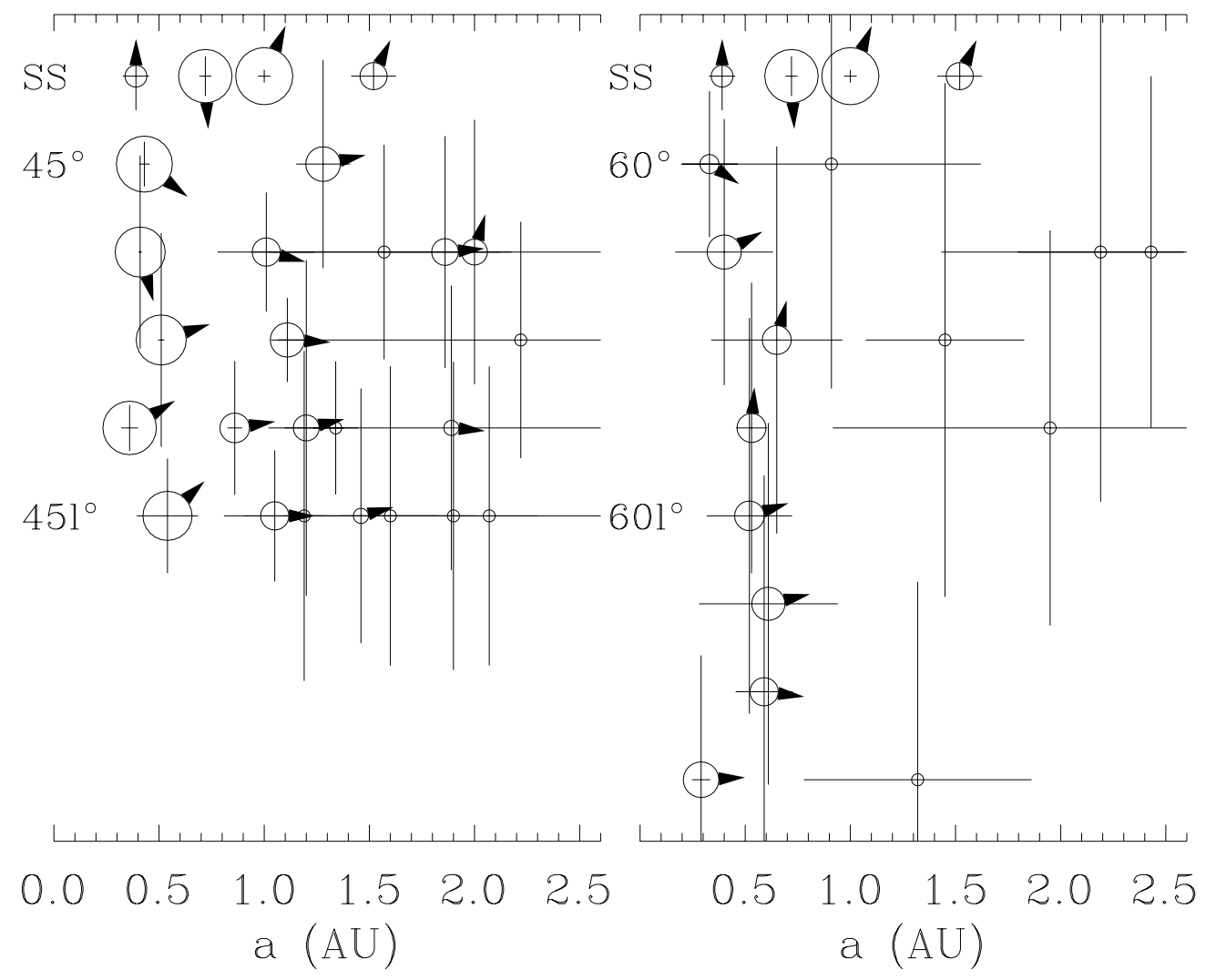

FIG. 9.-Final planetary states for our simulations with the disk centered around $\alpha$ Cen A inclined at $45^{\circ}$ or $60^{\circ}$ relative to the binary orbital plane. These high initial disk inclinations tend to truncate the radial extent of the circumstellar disk while causing large variations in the eccentricity and inclinations of most of the bodies. In the $45^{\circ}$ simulations, one large terrestrial planet formed near $\sim 0.5 \mathrm{AU}$, with smaller planets on highly inclined eccentric orbits that extend out to $\sim 2.2 \mathrm{AU}$. Most of the mass in the $60^{\circ}$ runs, however, was perturbed into the central star, and only one planet accreted mass in each system (all within $\sim 0.6$ $\mathrm{AU})$, while one to two additional planetesimals remained in orbit out to $\sim 2.5 \mathrm{AU}$. 


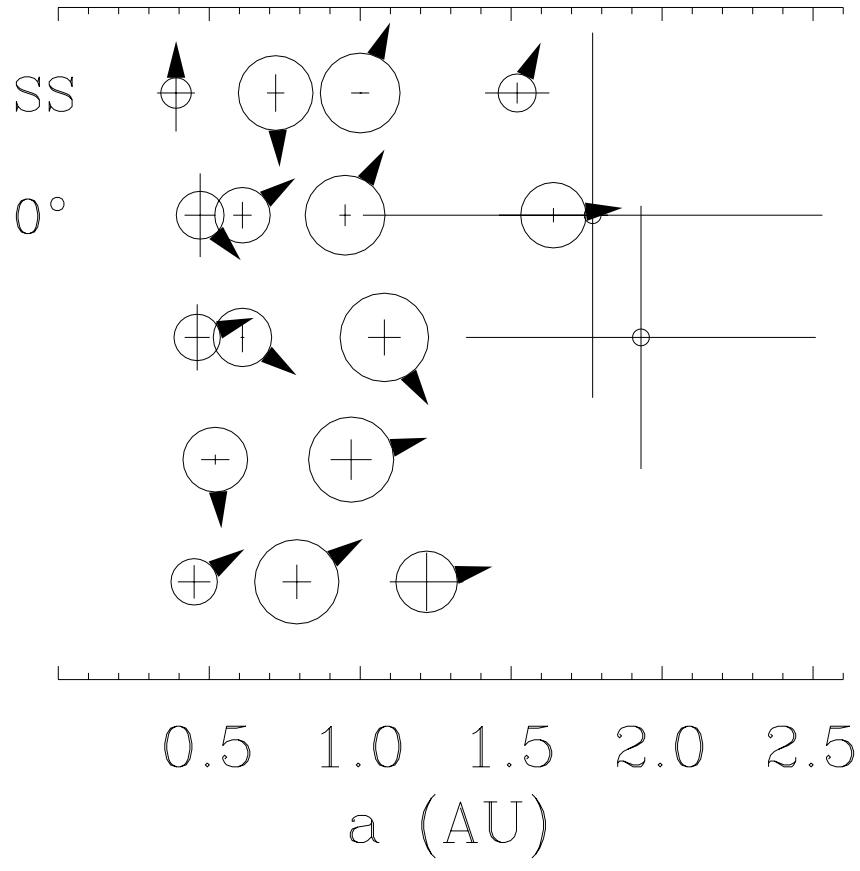

FIG. 10.-Final planetary states for our simulations with the disk centered around $\alpha \mathrm{Cen} \mathrm{B}$, with its initial inclination coplanar with the binary orbital plane. In these simulations, the more massive $\alpha$ Cen A truncates the circumstellar disk at a slightly smaller radius than in the converse case, and two to five planets are formed.

\section{DISCUSSION}

We have modeled the final stages of terrestrial planet formation in the $\alpha$ Cen binary star system. Our simulations all begin with (virtually) the same disk of planetesimals and planetary embryos. The distribution of planetary embryos and planetesimals in a binary star system is at present poorly constrained. Thus, the mass distribution of the circumstellar disk is adopted from simulations of terrestrial planet growth in our own solar system (including Jupiter and Saturn but no binary companion), which produced planetary configurations fairly similar to that actually observed (Chambers 2001). We ran dozens of simulations of planet formation around $\alpha$ Cen $\mathrm{A}$ with various inclinations of the planetesimal disk relative to the binary orbital plane, several runs with the disk around $\alpha \mathrm{Cen} \mathrm{B}$, and a few runs with the disk around a single star without giant planets for comparison purposes. Numerous smaller planetesimals were likely present at this stage of planetary growth in both the solar system and the $\alpha$ Cen system, but our calculations, like those of Chambers (2001) and of other groups, neglect these small bodies to make the problem computationally tractable. We also follow the example of solar system models in assuming that all physical collisions are completely inelastic; i.e., the bodies are merged together and never fragmented.

The binary companion $\alpha \mathrm{Cen} \mathrm{B}$ has a similar effect on terrestrial planet formation around $\alpha$ Cen $\mathrm{A}$ as Jupiter and Saturn have around the Sun, provided that the inclination of the circumstellar disk is $\leq 30^{\circ}$; i.e., the companion determines an outer boundary for the terrestrial region but does not prevent formation of terrestrial planets. The effect of the stellar companion became stronger as the disk inclination relative to the binary plane was increased. More than half of the initial mass in the disk was lost in all of the integrations in which $i=45^{\circ}$, and almost all of the mass (an average of $98 \%$ ) fell into the central star when $i=60^{\circ}$. When $i=180^{\circ}$, the binary companion has less influence on terrestrial planet formation than in the low disk inclination prograde case. These retrograde systems are more similar, however, to the lower disk inclination cases than to accretion simulations of a disk around the Sun with neither a companion nor giant planets in the system. Finally, $\alpha$ Cen A truncates the disk of accreting material a bit closer to $\alpha \mathrm{Cen} \mathrm{B}$ than in the converse case, as expected because of $\alpha$ Cen A's larger mass.

It is plausible that terrestrial planets with similar masses and orbits to those within our solar system may have formed in orbit about the star $\alpha$ Cen A and also about the star $\alpha$ Cen B, despite the proximity of these two stars. Would such planets be habitable? Probably not, if current models for the delivery of volatile materials to Earth (Morbidelli et al. 2000) are correct. These models suggest that the terrestrial planets received most of their volatiles from farther out in the solar system, principally from the asteroid belt. In the $\alpha$ Cen system, orbits within most of the region analogous to that in which Earth's volatiles are believed to have formed into planetesimals are unstable on rapid dynamical timescales. Thus, material would not be expected to exist in these zones, at least after the gas-dominated disk was dispersed; $\alpha$ Cen may thus have dry terrestrial planets, devoid of the $\mathrm{C} / \mathrm{H}_{2} \mathrm{O}$-based life that thrives on Earth.

It is our pleasure to thank Tony Dobrovolskis and Makiko Nagasawa for informative suggestions, which helped us improve the manuscript, and George Wetherill for a constructive referee's report. This research was supported in part by a NASA Graduate Student Researchers Program Fellowship awarded to E. V. Q.; J. E. C. is a NAS/ NRC Resident Research Associate at NASA Ames, whose work on this project was supported by the NASA Astrobiology Institute.

\section{APPENDIX}

\section{TERRESTRIAL PLANET GROWTH AROUND A SINGLE STAR LACKING GIANT PLANETS}

Giant planets analogous to those in the solar system cannot exist in the $\alpha$ Cen system (numerical integrations show that particles beyond 4 AU from each star cannot be stable; Wiegert \& Holman 1997; Chambers et al. 2002). Therefore, they are not included in our simulations of terrestrial planet growth in the $\alpha$ Cen system. For comparison, we performed three simulations of accretion in a disk around the Sun without giant planets perturbing the system. We used the same initial disk mass distribution and 7 day time step as used in most of our $\alpha$ Cen runs and followed the evolution of the disk around the Sun for up to 1 Gyr. In our first simulation, S_1, the radial extent of the disk ( $2.5 \mathrm{AU})$ within the first 5 Myr is similar to the $\alpha$ Cen simulations in which the circumstellar disk began at a low inclination relative to the binary orbital plane. As the integration progresses, more bodies remain in orbit farther from the Sun, reaching $\sim 3 \mathrm{AU}$ after $50 \mathrm{Myr}$. By the end of the $1 \mathrm{Gyr}$ integra- 


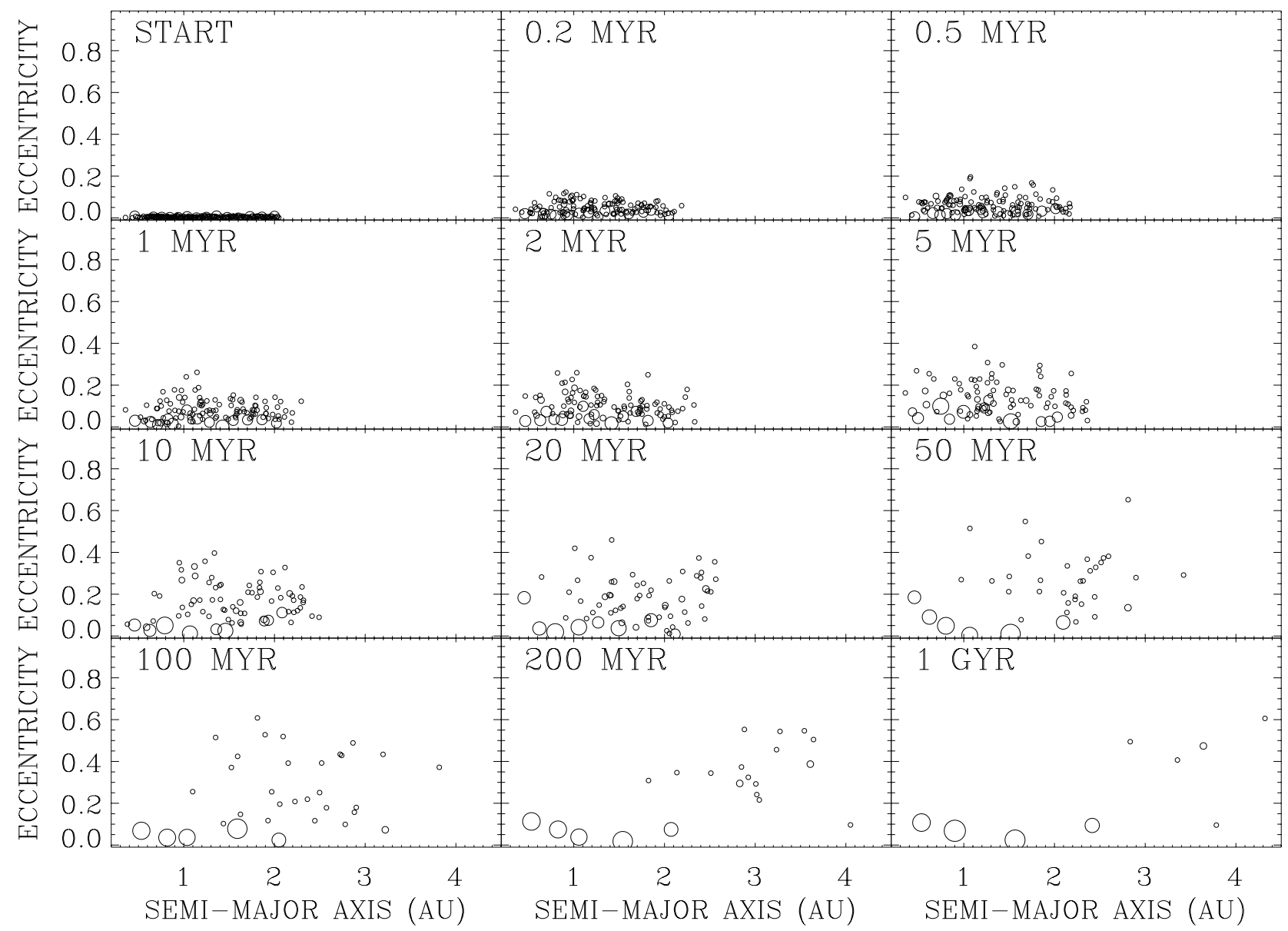

FIg. 11.-Evolution of the S_2 system is shown here. The lack of both a stellar companion and giant planets in this system allowed the disk to extend out farther from the central star than for simulations with a giant planet or a stellar companion in the system. The accretion rate was also slower in this system. Four planets formed within $2.5 \mathrm{AU}$ in all three simulations, while minor planets remained in orbit even beyond $4 \mathrm{AU}$ of the Sun. Three planets that are in orbit beyond $5 \mathrm{AU}$ of the Sun are not shown in this figure.

tion, four planets have formed within 2.3 AU, and nine minor planets still orbit the Sun between 1.3 and 4.8 AU. All of the initial disk mass remained in this system, with the exception of the ejection of one planetesimal at $342 \mathrm{Myr}$.

For our second accretion simulation around the Sun, S_2, we shifted the initial mean anomaly of a planetesimal near 1 AU by $1 \mathrm{~m}$ and started the integration with all other initial conditions identical to the $\mathrm{S} \_1$ simulation (Fig. 11). In this case, the radial extent of the disk was similar to the $\mathrm{S} \_1$ run throughout the simulation. All of the initial mass in the disk remained in orbit around the Sun for the first $305 \mathrm{Myr}$, and only three minor planets were ejected by 1 Gyr. Four planets formed in this system within 2.4 AU, while eight minor planets remained in orbit between 2.9 and 8.2 AU. An additional minor planet remained in the system at $a \sim 43 \mathrm{AU}$ but on an extremely eccentric orbit $(e=0.98)$.

Because there is not a massive body (besides the central star) in these systems, the usual removal mechanisms (close encounters, secular excitation of eccentricities and inclinations, and resonances involving a second massive body) do not exist. Therefore, most of the mass remains in orbit and the accretion rate was much slower than for the $\alpha$ Cen simulations described in this

TABLE 2

Statistics for Accretion around Sun with neither Giant Planets nor a Binary Companion

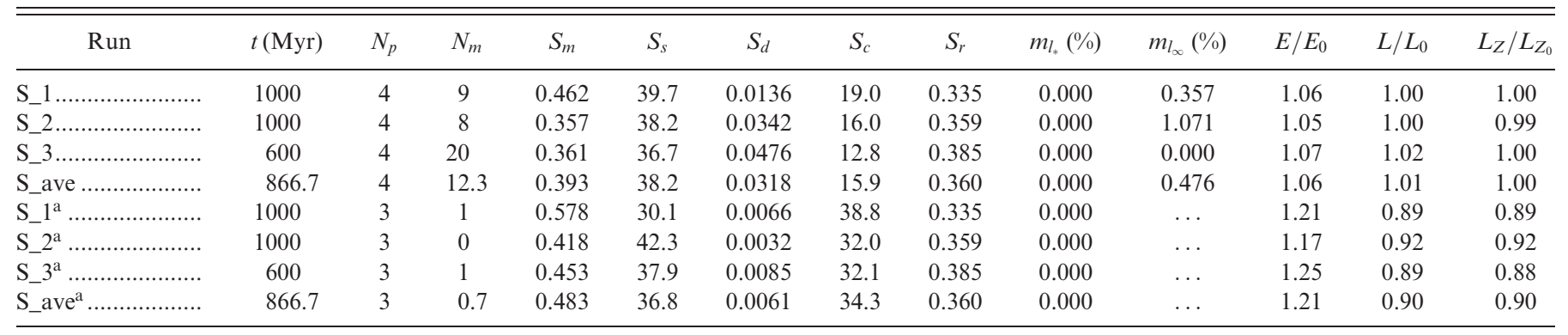

a Most of the planets that remain in orbit beyond $2 \mathrm{AU}$ of the Sun at the end of the simulations are on highly eccentric orbits. Thus, the statistics for planets that remain within $2 \mathrm{AU}$ are given in the bottom half of this table for better comparison with the statistics of the final systems in the $\alpha$ Cen simulations. 


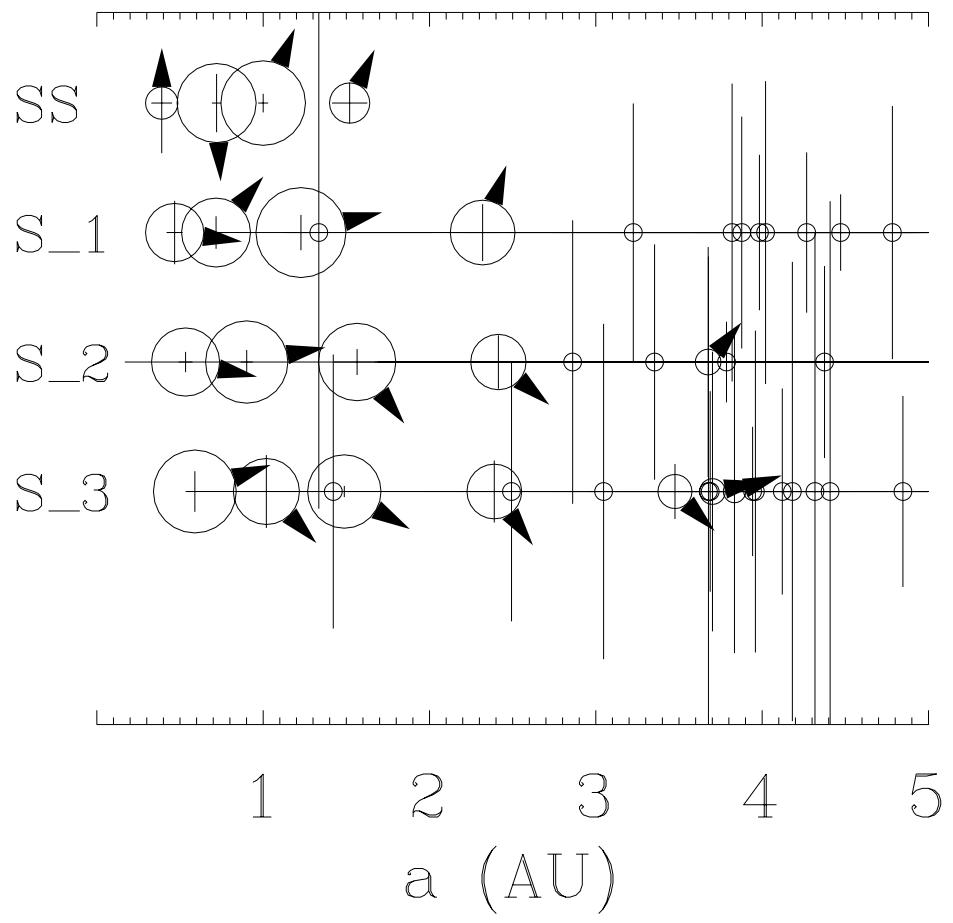

FIG. 12.-Final planetary states for our accretion simulations around the Sun, with neither giant planets nor a stellar companion perturbing the system. The radius of each symbol is proportional to the radius of the final planet that it represents, the horizontal line through each body signifies the periastron and apastron distances, the vertical line represents the inclinations, and the arrow indicates the final spin orientation of each planet. In these simulations, $100 \%$ of the initial disk mass remained in the system for at least $300 \mathrm{Myr}$. Note the wider range of semimajor axis compared with Figs. 8-10. In the S 2 simulation, three planetesimals remain in orbit beyond $5 \mathrm{AU}$ of the Sun and are omitted from this figure: one at $5.85 \mathrm{AU}$ with an eccentricity of $e=0.71$, one at 8.19 AU with $e=0.57$, and another at $43.33 \mathrm{AU}$ with $e=0.98$. Five planetesimals in the S_3 run are also not shown in this figure and are located at the following semimajor axes: 5.14 $\mathrm{AU}(e=0.50), 5.88 \mathrm{AU}(e=0.52), 5.93 \mathrm{AU}(e=0.42), 7.19 \mathrm{AU}(e=0.92)$, and $17.36 \mathrm{AU}(e=0.93)$.

paper. Our third simulation began with the same initial circumstellar disk but with the mean anomaly of the planetesimal closest to $1 \mathrm{AU}$ shifted by $2 \mathrm{~m}$ along its orbit. In this case, all of the mass remained in the system throughout the $600 \mathrm{Myr}$ integration. Figure 12 shows the final planetary systems for our accretion simulations centered around the Sun, with the first row (SS) referring to the four terrestrial planets in the solar system. The radius of each body is proportional to the radius of the planet that it represents, the horizontal lines through each body indicate the periastron and apastron distances to the central star, the vertical lines represent the inclination relative to the initial disk orbital midplane, while the arrows show the orientation of the final spin axes of each planet (a downward arrow implies retrograde spin). In all three simulations, four planets more massive than Mercury formed within $2.5 \mathrm{AU}$ of the Sun, while additional minor planets remained in the systems (most of which orbited the Sun beyond 2.5 AU). Table 2 gives the statistics (as described in $\S 4.2$ ) for these simulations, followed by the statistics for the final planets that remain within $2 \mathrm{AU}$ of the Sun in these simulations. Aside from the amount of mass lost, these truncated systems (of planets that orbit within $2 \mathrm{AU}$ of the Sun) look like systems formed with giant planet or binary companions.

Abt, H. A. 1977, Sci. Am., 236, 96

Akeson, R. L., Koerner, D. W., \& Jensen, E. L. N. 1998, ApJ, 505, 358

Bate, M. R. 2000, MNRAS, 314, 33

Bodenheimer, P., Burkert, A., Klein, R. I., \& Boss, A. P. 2000, in Protostars and Planets IV, ed. V. Mannings, A. P. Boss, \& S. S. Russell (Tucson: Univ. Arizona Press), 675

Butler, R. P., Marcy, G. W., Williams, E., Hauser, H., \& Shirts, P. 1997, ApJ, 474, L115

Chambers, J. E. 1999, MNRAS, 304, 793 2001, Icarus, 152, 205

Chambers, J. E., Quintana, E. V., Duncan, M. J., \& Lissauer, J. J. 2002, AJ, 123,2884

Chambers, J. E., Wetherill, G. W. \& Boss, A. P. 1996, Icarus, 119, 261

Cochran, W. D., Hatzes, A. P., Butler, R. P., \& Marcy, G. W. 1997, ApJ, 483,457

Endl, M., Kurster, M., Els, S., Hatzes, A. P., \& Cochran, W. D. 2001, A\&A, 374, 675

Greenberg, R., Hartmann, W. K., Chapman, C. R., \& Wacker, J. F. 1978, Icarus, 35,1

Hale, A. 1996, in Circumstellar Habitable Zones: Proc. First Int. Conf., ed. L. R. Doyle (Menlo Park: Travis House Publications), 143

Innanen, K. A., Zheng, J. Q., Mikkola, S., \& Valtonen, M. J. 1997, AJ, 113, 1915
REFERENCES

Kokubo, E., \& Ida, S. 1998, Icarus, 131, 171

Kortenkamp, S. J. \& Wetherill, G. W. 2000, Icarus, 143, 60

Kortenkamp, S. J., Wetherill, G. W., \& Inaba, S. 2001, Science, 293, 1127

Kozai, Y. 1962, AJ, 67, 591

Lissauer, J. J. 1993, ARA\&A, 31, 129

Lubow, S. H., \& Artymowicz, P. 2000, in Protostars and Planets IV, ed. V. Mannings, A. P. Boss, \& S. S. Russell (Tucson: Univ. Arizona Press), 731

Marzari, F., \& Scholl, H. 2000, ApJ, 543, 328

Mathieu, R. D., Ghez, A. M., Jensen, E. L. N., \& Simon M. 2000, in Protostars and Planets IV, ed. V. Mannings, A. P. Boss, \& S. S. Russell (Tucson: Univ. Arizona Press), 703

Morbidelli, A., Chambers, J., Lunine, J. I., Petit, J. M., Robert, F., Valsecchi, G. B., \& Cyr, K. E. 2000, Meteoritics Planet. Sci., 35, 1309

Öpik, E. J. 1951, Proc. R. Irish Acad., 54, 165

Rodriguez, L. F., et al. 1998, Nature, 395, 355

Safronov, V. S. 1969, Evolution of the Protoplanetary Cloud and Formation of the Earth and the Planets (Moscow: Nauka)

Wetherill, G. W., \& Stewart, G. R. 1989, Icarus, 77, 330

Wiegert, P. A., \& Holman, M. J. 1997, AJ, 113, 1445

Wisdom, J., \& Holman, M. 1991, AJ, 102, 1528 